\title{
THE GLOBULAR CLUSTER SYSTEM OF NGC 1399. III. VLT SPECTROSCOPY AND DATABASE ${ }^{1}$

\author{
B. Dirsch, ${ }^{2}$ T. Richtler,${ }^{2}$ D. Geisler,${ }^{2}$ K. Gebhardt,${ }^{3}$ M. Hilker,${ }^{4}$ M. V. Alonso, ${ }^{5}$ J. C. Forte, ${ }^{6}$ E. K. Grebel, ${ }^{7}$ \\ L. Infante, ${ }^{8}$ S. Larsen, ${ }^{9}$ D. Minniti, ${ }^{8}$ and M. Rejkuba ${ }^{9}$ \\ Received 2003 January 31; accepted 2003 October 7
}

\begin{abstract}
Radial velocities of 468 globular clusters around NGC 1399, the central galaxy in the Fornax cluster, have been obtained with FORS2 and the Mask Exchange Unit (MXU) at the ESO Very Large Telescope. This is the largest sample of globular cluster velocities around any galaxy obtained so far. The mean velocity uncertainty is $50 \mathrm{~km} \mathrm{~s}^{-1}$. This data sample is accurate and large enough to be used in studies of the mass distribution of NGC 1399 and the properties of its globular cluster system. Here we describe the observations and the reduction procedure, and we discuss the uncertainties of the resulting velocities. The complete sample of cluster velocities that is used in a dynamical study of NGC 1399 is tabulated. A subsample is compared with previously published values.
\end{abstract}

Key words: dark matter — galaxies: elliptical and lenticular, cD — galaxies: halos — galaxies: individual (NGC 1399) — galaxies: kinematics and dynamics — galaxies: star clusters

\section{INTRODUCTION}

In the context of understanding the distribution of dark matter in galaxies, early-type galaxies in the centers of galaxy clusters play an important role. In these places the highest dark matter densities are found and, accordingly, the transition between luminous and dark matter-dominated regions can be best investigated. In the inner regions of elliptical galaxies this can be done by long-slit spectroscopy measuring integrated spectral line profiles (see Kronawitter et al. 2000) and Gerhard et al. (2001) for a large sample of galaxies).

In the outer region of an elliptical galaxy-in an area where dark matter begins to dominate - this approach is hardly feasible because of the low surface brightness. Globular clusters (GCs) and planetary nebulae (PNe), which can be traced to large radii, are the best tools to study the dynamics at larger radii. However, the distribution of radial velocities of particles is degenerate with respect to the galaxy mass profile and the orbital structure of the dynamical probes (e.g., Merritt \& Tremblay 1993). If no other constraints on the velocity dispersion tensor and mass distribution are available, then radial velocities for several hundred or more clusters are required to

\footnotetext{
1 Based on observations collected at the European Southern Observatory, Cerro Paranal, Chile; ESO program 66.B-0393.

2 Departamento de Física, Universidad de Concepción, Casilla 160-C, Concepción, Chile; bdirsch@cepheid.cfm.udec.cl.

${ }^{3}$ Department of Astronomy, University of Texas at Austin, Austin, TX 78712 .

${ }^{4}$ Sternwarte der Universität Bonn, Auf dem Hügel 71, D-53121 Bonn, Germany.

5 Observatorio Astronómico de Córdoba, Laprida 854, Córdoba, 5000, CONICET, Argentina CNRS UMR 5572; Observatoire Midi-Pyrénées, 14 Avenue Edouard Belin, F-31400 Toulouse, France.

${ }^{6}$ Facultad Ciencias Astronomicas y Geofisicas, Universidad Nacional de La Plata, Paseo del Bosque S/N, 1900-La Plata, UNLP and CONICET, Argentina.

7 Max-Planck-Institut für Astronomie, Königstuhl 17, D-69117, Heidelberg, Germany.

${ }^{8}$ Departamento de Astronomía y Astrofísica, Pontificia Universidad Católica, Vicuña Mackenna 4860 Santiago 22, Chile.

9 European Southern Observatory, Karl-Schwarzschild-Strasse 2, D-85748 Garching, Germany.
}

break this degeneracy (e.g., Merritt 1993). Therefore, if we want to lift this degeneracy, we are limited to those nearby large elliptical galaxies with sufficiently populous cluster systems. M87 is such a candidate in the northern hemisphere. Cohen \& Ryzhov (1997) observed about 200 radial velocities using the Keck telescope. This sample has been improved and enlarged to about 350 velocities (Côté et al. 2001; Hanes et al. 2001).

Another promising target is the central galaxy of the Fornax cluster, NGC 1399. Its populous globular cluster system (GCS) has been investigated by Hanes \& Harris (1986), Bridges et al. (1991), Wagner et al. (1991), Ostrov et al. (1993), KisslerPatig et al. (1997), Grillmair et al. (1999), and most recently by Dirsch et al. (2003, hereafter Paper I), the latter covering a field of $36^{\prime} \times 36^{\prime}$. The GCS has been the target of three spectroscopic studies. Grillmair et al. (1994) obtained lowdispersion spectra of $47 \mathrm{GCs}$ within a radial distance of $9^{\prime}$ around NGC 1399 with the Low Dispersion Survey Spectrograph on the Anglo-Australian Telescope (AAT). A typical uncertainty of their velocities is $150 \mathrm{~km} \mathrm{~s}^{-1}$. Another contribution comes from Minniti et al. (1998) who used the ESO Multi-Mode Instrument at the ESO $3.5 \mathrm{~m}$ New Technology Telescope (NTT) to measure velocities of 19 GCs. Their velocity uncertainties are also large: the mean uncertainty is $128 \mathrm{~km} \mathrm{~s}^{-1}$. The velocities with the smallest errors available so far are obtained by Kissler-Patig et al. (1998) with the Low Resolution Imaging Spectrograph at the Keck telescope. They determined the velocities of $21 \mathrm{GCs}$ with an average uncertainty of $45 \mathrm{~km} \mathrm{~s}^{-1}$. Kissler-Patig et al. (1999) compiled the velocity determinations from these three studies, ending up with $74 \mathrm{GC}$ velocities.

Beside the GCs, PNe have been used for dynamical studies of NGC 1399 by Arnaboldi et al. (1994) and Napolitano et al. (2002). Both studies used the same database of 37 PN velocities in the inner $4^{\prime}$ of NGC 1399. At smaller radial distances $\left(\leq 97^{\prime \prime}\right)$ the galaxy light itself has been employed to constrain dynamical models by Saglia et al. (2000), Kronawitter et al. (2000), and Gerhard et al. (2001).

To greatly improve our knowledge of its GCS kinematics and galaxy halo dynamics, we used the FORS2 at the VLT to obtain spectra of globular clusters out to a radial distance of $8^{\prime}$, 


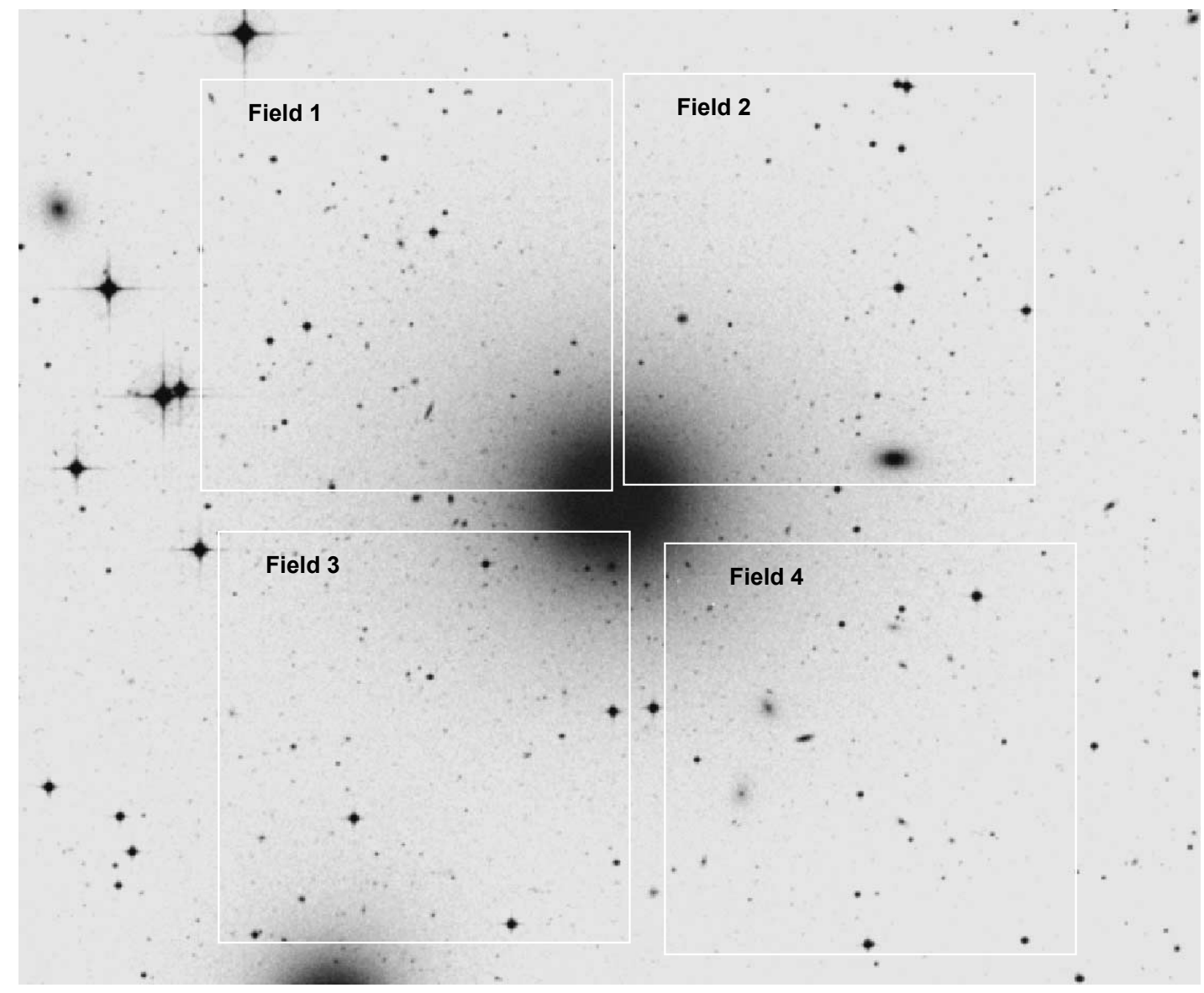

FIG. 1.-Positions of the four fields (each having a size of $7^{\prime} \times 7^{\prime}$ ) in which we obtained the spectra overlaid on a DSS image (blue filter). East is to the left, north to the top; the size of the image is $19.3 \times 15$. $^{\prime} 6$.

which corresponds to approximately $45 \mathrm{kpc}$. A distance modulus of $m-M=31.4(19 \mathrm{Mpc}$ ) is used (see Dirsch et al. 2003 and discussion therein).

The observations aim at a significant improvement in the number and quality of dynamical probes.

The selected cluster candidates have luminosities brighter than $V=22.5 \mathrm{mag}$. An ideal telescope to obtain the required spectra of such faint objects in the southern hemisphere is the ESO Very Large Telescope. A spectroscopic resolution of approximately $3 \AA$ is desirable for our task, and thus FORS2 with the Mask Exchange Unit (MXU) is an appropriate instrument since it allows the simultaneous observation of some 100 objects.

In this article we present the data analysis and error discussion of our measurements. The complete data set of 502 cluster velocities is presented, which is the basis of the dynamical analysis published in Richtler et al. (2003, hereafter Paper II). These papers are part of a bigger effort to study the dynamics and stellar content of NGC 1399, which also includes new photometric wide-field observations published in Paper I.

\section{SPECTROSCOPIC MASK PREPARATION}

The preparation of the slit masks we used for observing NGC1399 at the VLT is an important step in the overall investigation. Hence, we describe this first step in detail before discussing the observations themselves. Our candidate GCs have been selected on the basis of new wide-field photometry in Washington $C$ and Kron-Cousins $R$ of the cluster system of NGC 1399, obtained at the Cerro Tololo Inter-American Observatory (CTIO) $4 \mathrm{~m}$ MOSAIC system. For a description of the photometric observations the reader is referred to Paper I.

For the point-source selection we used a color criterion $(0.9<(C-T 1)<2.4)$ and also a morphological criterion based on the SExtractor class (Bertin \& Arnout 1996); only objects with class larger than 0.8 are retained, based on artificial star experiments and comparison with the VLT preimages. This morphological selection limited our sample to objects brighter than $R=23.3 \mathrm{mag}$, which allows us to obtain the required signal-to-noise ratio $(\mathrm{S} / \mathrm{N})$ in the spectra.

For accurate slit placement on the MXU masks, preimaging is required. Service mode observations with FORS2 at the VLT in 2000 October provided us with four FORS2 fields, each of them $7^{\prime} \times 7^{\prime}$, arranged in a quadratic configuration (see Fig. 1). Each field has been exposed for $30 \mathrm{~s}$ in Johnson $V$. We identified the selected clusters in these images and placed the slits accordingly. Since the VLT images had superior seeing (around 0.6), compared with our MOSAIC observations, we used them to check the starlike appearance of the selected objects. This left us with about 500 globular cluster candidates.

A major point during the preparation of the masks, which was done with the ESO FORS Instrumental Mask Simulator (FIMS), ${ }^{10}$ were the choices of the slit width and particularly

\footnotetext{
${ }^{10}$ See http://http.hq.eso.org/observing/p2pp/OSS/FIMS/FIMS-tool.html.
} 

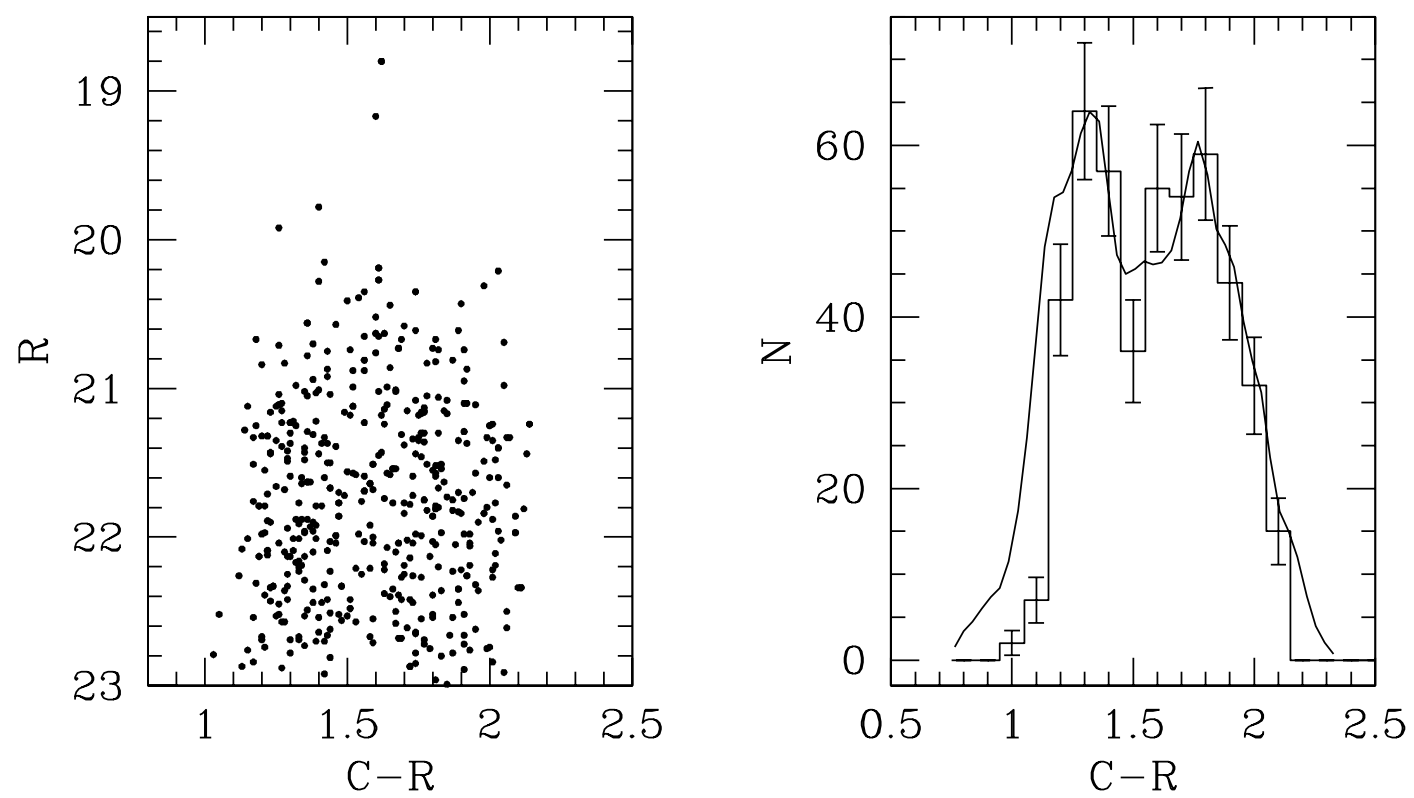

FIG. 2.-Left: Color-magnitude diagram of the final cluster sample. Right: Distribution of the final sample (histogram) is compared with the arbitrarily scaled, adaptively smoothed (Epanachikov kernel) color distribution of the whole MOSAIC sample (Paper I) within the common radial range. The agreement between the two samples is very good and indicates that the spectroscopic sample is not biased.

the slit lengths. We decided for a slit width of $1^{\prime \prime}$, which turned out to be appropriate for the normally subarcsecond seeing conditions.

The slit length was a more complicated issue. The spectra of our fainter targets are sky-dominated, and the sky subtraction is best done by measuring object and sky in the same slit. However, the severe disadvantage with slits of the appropriate length, $5^{\prime \prime}$ or longer, is that they would cover up many objects that otherwise would have been observable with smaller length, which runs contrary to our objective of observing as many targets as possible. Moreover, the use of a longer slit considerably lowers the flexibility of choosing slit positions according to the random location of targets with a high surface number density in a mask. Therefore, we decided to set sky slits independently from the object slits and to subtract the sky after the wavelength calibration, trusting in its accuracy.
For most slits, the size then was $1^{\prime \prime} \times 2^{\prime \prime}$, being $1^{\prime \prime} \times 5^{\prime \prime}$ only for the few bright objects (normally foreground stars), which were needed to accurately position the mask. In this manner, we could fill a mask with up to 120 slits, typically 40 cluster candidates, 40 sky slits, and many miscellaneous objects, among them other point sources not matching our strict selection criteria, or background galaxies.

The color-magnitude diagram of the final sample for which we obtained velocities is shown in Figure 2 together with its color distribution that is compared with the color distribution of the total MOSAIC sample within the same radial range. This figure illustrates that the final sample is not subject to color selection effects.

\section{THE OBSERVATIONS}

The observations were performed during the period from 2000 November 30 to December 2 at the European Southern

TABLE 1

Observed Masks, Their Central Positions, and Exposure Times

\begin{tabular}{|c|c|c|c|}
\hline Field & Mask No. & Center Position & $\begin{array}{c}t_{\exp } \\
\text { (minutes) }\end{array}$ \\
\hline 1 & 75 & $3: 38: 54-35: 23: 30$ & 90 \\
\hline 1 & 76 & $3: 38: 45-35: 23: 30$ & 45 \\
\hline 1 & 77 & $3: 38: 35-35: 23: 27$ & 45 \\
\hline 1 & 78 & $3: 38: 45-35: 25: 08$ & 90 \\
\hline 2 & 80 & $3: 38: 21-35: 23: 30$ & 90 \\
\hline 2 & 81 & $3: 38: 08-35: 23: 31$ & 45 \\
\hline 2 & 82 & $3: 38: 11-35: 25: 25$ & 45 \\
\hline 3 & 84 & $3: 38: 54-35: 31: 00$ & 45 \\
\hline 3 & 86 & $3: 38: 37-35: 30: 59$ & 90 \\
\hline 4 & 89 & $3: 38: 17-35: 31: 01$ & 45 \\
\hline 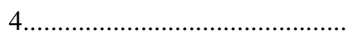 & 90 & $3: 38: 13-35: 31: 01$ & 90 \\
\hline 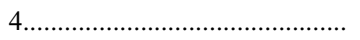 & 91 & $3: 38: 01-35: 31: 01$ & 90 \\
\hline 4 & 92 & $3: 38: 08-35: 29: 16$ & 45 \\
\hline
\end{tabular}

NoTE.-Units of right ascension are hours, minutes, and seconds, and units of declination are degrees, arcminutes, and arcseconds. 
TABLE 2

Summary of Number and Type of Observed Objects on Each Mask

\begin{tabular}{|c|c|c|c|c|c|}
\hline $\begin{array}{l}\text { Mask No. } \\
\text { (1) }\end{array}$ & $\begin{array}{c}\text { Candidates } \\
\text { (2) }\end{array}$ & $\begin{array}{l}\text { Other Point } \\
\text { Sources } \\
\text { (3) }\end{array}$ & $\begin{array}{c}\text { Galaxies } \\
\text { (4) }\end{array}$ & $\begin{array}{l}\text { Sky Slits } \\
\text { (5) }\end{array}$ & $\begin{array}{c}\text { Obtained } \\
\text { Velocities } \\
\text { (6) }\end{array}$ \\
\hline 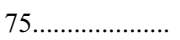 & 32 & 11 & 16 & 51 & 27 \\
\hline 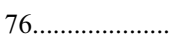 & 45 & 12 & 16 & 47 & 40 \\
\hline 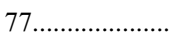 & 42 & 14 & 6 & 59 & 37 \\
\hline 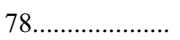 & 32 & 29 & 10 & 45 & 37 \\
\hline 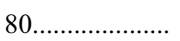 & 38 & 19 & 4 & 54 & 49 \\
\hline 81..................... & 39 & 15 & 16 & 37 & 42 \\
\hline $82 \ldots$ & 46 & 5 & 8 & 27 & 36 \\
\hline 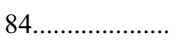 & 35 & 6 & 31 & 34 & 26 \\
\hline 86.................... & 61 & 4 & 6 & 39 & 59 \\
\hline $89 \ldots \ldots \ldots \ldots \ldots$ & 50 & 13 & 13 & 28 & 48 \\
\hline 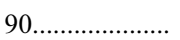 & 28 & 28 & 14 & 34 & 43 \\
\hline 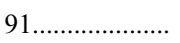 & 32 & 17 & 16 & 44 & 29 \\
\hline 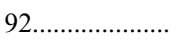 & 22 & 16 & 19 & 40 & 29 \\
\hline Total ............. & 502 & 189 & 175 & 539 & 502 \\
\hline
\end{tabular}

Notes.-Cols. (2), (3), (4): number of objects for which we obtained a spectrum. Col. (6): number of clusters for which a velocity has been determined.

Observatory at Cerro Paranal with the Very Large Telescope facility. The telescope was UT2 (Kueyen) and the instrument the focal reducer FORS2, equipped with the MXU. The detector was a SiTE SI-424A backside thinned CCD with a pixel size of $24 \times 24 \mu \mathrm{m}$. The total field of view is $6.83 \times 6{ }^{\prime} 83$. The observations are summarized in Table 1 . The four VLT fields in which the masks were placed are shown overlaid on a DSS image of NGC 1399 in Figure 1.

We used the 600B grism without filter, which provides a resolution of around $2.5 \AA$. The spectral range covered was about $2000 \AA$. However, as a result of the position of a given slit in the mask, the limiting short and long wavelengths varied considerably, ranging from 3500 to $6500 \AA$. In most cases the grism efficiency degraded the $\mathrm{S} / \mathrm{N}$ shortward of $3800 \AA$ drastically, so this region could not be used. Mask 89 has been observed using the $300 \mathrm{~V}$ grism with a lower spectral resolution $\left(110 \AA \mathrm{mm}^{-1}\right)$. This has been done to see the effect of using a certain grism. The analysis showed no difference in the quality of the derived velocities for the two grisms.

We exposed 13 masks (exposure times were either 45 minutes or $2 \times 45$ minutes). The seeing was always subarcsecond and ranged from 0 . 6 to $00^{\prime \prime} 9$. Flat fielding was done with standard lamp flats. For the wavelength calibration, a $\mathrm{HeHgCd}$ lamp was exposed during service day-time calibration.

The total number of spectra obtained is 1462 . This sample is composed of 531 sky spectra (see Table 2), 512 spectra of cluster candidates, 190 spectra of point sources of unknown nature at the time of mask preparation (stars, unresolved galaxies, or globular clusters), 176 galaxies, and 53 "bright" objects, mainly stars, which were needed to adjust the mask. Since some objects (about 80) were observed in two different masks to assess the velocity uncertainties, the total number of objects is smaller by this number than the number of spectra. There are two reasons why we found clusters in the (randomly selected) point-source sample: first, the MOSAIC data is not dithered, and thus for a considerable fraction of clusters no photometry was obtained. Second, our employed morphological selection criteria were very strict, and the completeness of faint cluster identification was rather low (see also Paper I).

Table 1 lists the relevant data of the observations, starting with the number of the respective FORS field, the mask number, the center coordinates (J2000.0), the exposure time (90 minutes means that two exposures of 45 minutes have been stacked).

\section{THE DATA REDUCTION}

The basic reduction (bias subtraction, flat fielding, trimming) has been done with standard procedures within IRAF. For the later extraction of the spectra the APEXTRACT package has been used.

Regarding the removal of cosmic rays, we found after some experimenting that the task FILTER/COSMICs under MIDAS gave the most satisfactory results. A few artefacts remained in spite of that, best visible in the faint spectra.

In the flat-fielded image the spectra have a separation of only a few pixels and are curved along the dispersion axis, which has to be fitted before they can be extracted. The curvature is strongest at the frame edges, where the deviation from the center to the edge is approximately 6 pixels. We traced each spectrum along the dispersion axis on the flatfield image of the mask because of their much clearer signal. We then kept the tracing parameters and optimized the size of the apertures with the goal of minimizing the sky contribution in the object spectra that has been done on the science spectra. The final sizes of the apertures depend on the seeing and varies between $1^{\prime \prime}$ and 1".4. On some masks a few spectra overlap, and they have been excluded from further analysis.

We employed the IRAF task IDENTIFY to calibrate both object and sky spectra. Typically, around $18 \mathrm{He}, \mathrm{Hg}$, and $\mathrm{Cd}$-lines were kept for the line list. The calibration uncertainty is $\pm 0.04 \AA$.

The bright O I skyline at $5567 \AA$, which is present in all spectra, can be used to correct zero-point differences between the masks. Differences up to $\pm 0.8 \AA$ have been found. The reason for these systematic differences is the uncertainty in the mask placement, which is cited in the MXU manual ${ }^{11}$ to be $13 \mu \mathrm{m}$. This is approximately half a pixel, or $0.6 \AA$, using the

\footnotetext{
11 See http://www.eso.org/instruments/fors/userman.
} 
600B grism and thus is in good agreement with our measured zero-point differences.

Within a mask the position of the $\mathrm{O}$ I skyline was constant to within $0.04 \AA$ consistent with the calibration uncertainty. An exception is mask No. 89 (with the grism 300V), where we found a systematic linear increase/decrease of the $\mathrm{O}$ I line position from 5566.3 to $5567.91 \AA$. We corrected for this with a linear interpolation in the wavelength calibration. The systematic behavior in mask No. 89 is probably due to a slight rotation of the mask.

For the sky subtraction, we used spectra that were observed through slits placed in empty sky areas. For each object adjacent sky spectra within a certain radial distance from NGC 1399 have been selected to attempt to obtain the best sky spectrum. The radial width of these annuli had been chosen to be smaller near to NGC 1399 and wider further away (1.'2 for distances smaller than 2.5 and 1.7 for larger distances). Typically, three to 11 sky spectra were averaged and subtracted from the object spectra. The quality of the subtraction was judged by examining the residuals around the bright skylines in the red.

This procedure resulted in good sky-subtracted spectra as long as wavelengths longer than $4000 \AA$ are considered. For shorter wavelengths, the uncertainties in sensitivity ("flat-field uncertainties") between sky and object spectra make the sky subtraction difficult. However, because of the low $\mathrm{S} / \mathrm{N}$ of the spectra blueward of $4000 \AA$, this uncertainty is not important anyway. Some example spectra around $T 1=21$ are shown in Figure 3.

\section{VELOCITIES}

\subsection{Velocity Determination}

We determined velocities using two different techniques: first, by using the correlation with an object of known velocity and, second, by measuring the redshift of an object directly with identified absorption lines.

The line measurements were performed with the help of the RVIDLINES task within IRAF. Typically, around 15 features have been fitted for the velocity determinations.

For the correlation technique, we used the task FXCOR implemented in IRAF (the technique is described by Tonry \& Davis 1979). We did not use any Fourier filtering, instead smoothing very noisy spectra with a median filter (3 pixels). The continuum has been subtracted by FXCOR with a spline fit to the spectra and a $2 \sigma$ clipping algorithm around the fit line. The range that has been proved to be best suited for the velocity determination is 4500-5500 $\AA$. However, for fainter objects, we adjusted the range individually to find the most significant correlation peak. As a template, we used a high $\mathrm{S} / \mathrm{N}$ spectrum (S/N about 40) of NGC 1396, a small galaxy with low intrinsic velocity dispersion and a spectrum similar to that of a cluster. This object is on mask No. 82, which accordingly served as the reference mask. Zero-point differences with the other masks had been accounted for by using the position of the sky O I-line. We determined NGC 1396's velocity by measuring lines and obtained $815 \pm 8 \mathrm{~km} \mathrm{~s}^{-1}$ as the heliocentric velocity. This value is considerably lower than most older values from the literature: da Costa et al. (1998) found $856 \pm 37 \mathrm{~km} \mathrm{~s}^{-1}$ and in the RC3 catalog $894 \pm 29 \mathrm{~km}$ $\mathrm{s}^{-1}$ is given (de Vaucouleurs et al. 1991). However, Drinkwater et al. (2001b) quote $808 \pm 8 \mathrm{~km} \mathrm{~s}^{-1}$. The mean velocity of all clusters is $1438 \pm 15 \mathrm{~km} \mathrm{~s}^{-1}$. As for NGC 1399 itself, consulting the NASA/IPAC Extragalactic Database, one

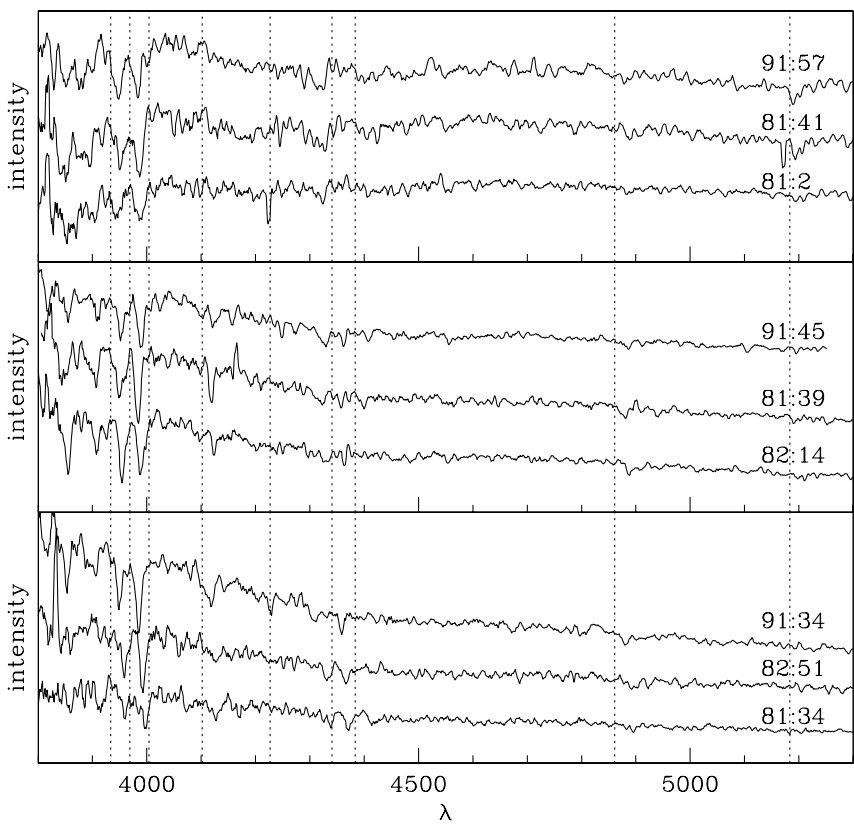

Fig. 3.-Example spectra of brighter cluster candidates (smoothed by $3.6 \AA$ for illustration) in three color regimes. Top: $C-T 1 \approx 1.95$; middle: $C-T 1 \approx$ 1.35 ; bottom: $C-T 1 \approx 1.15$. We indicated the rest-frame wavelengths of some prominent absorption features by the dotted lines. Let to right: Ca K $\lambda 3933.66$, $\mathrm{Ca} \mathrm{H} \lambda 3968.47, \mathrm{Fe}_{\mathrm{I}} \lambda 4045.81, \mathrm{H} \delta \lambda 4101.73, \mathrm{Ca}$ I and $\mathrm{Fe}$ I blend $\lambda 4226.73$, $\mathrm{H} \gamma \lambda 4340.46, \mathrm{Fe}$ I $\lambda 4383.54, \mathrm{H} \beta \lambda 4861.32, \mathrm{Mg}$ I $\lambda 5183.64$. The number at the right side of the spectra is the identifier of the objects explained in $\S 5.3$ and used in Table 3.

finds nine measurements of its radial velocity with quoted uncertainties. After skipping two of them (one has a discrepant value and the other a large uncertainty of $200 \mathrm{~km} \mathrm{~s}^{-1}$ ), the weighted mean value is $1442 \pm 9 \mathrm{~km} \mathrm{~s}^{-1}$. Our mean radial velocity of the entire cluster sample is $1438 \pm 15 \mathrm{~km} \mathrm{~s}^{-1}$, so we are confident of our absolute velocity calibration.

\subsection{Velocity Uncertainties}

The differences between cluster velocities that were derived with individual line and with correlation measurements (shown in Fig. 4) can be used to study the velocity uncertainties. A dependence of the differences on the brightness of the objects is expected and can be observed. However, deviations as large as $500 \mathrm{~km} \mathrm{~s}^{-1}$ are more than $3 \sigma$ larger than what is expected from the measurement uncertainties. This indicates that at least for some measurements, systematic errors dominate over statistical errors. We will show later that errors in the line measurements of noisy spectra are responsible for this behavior. The mean value of the difference between line and correlation measurements is $-6.2 \pm 0.3 \mathrm{~km} \mathrm{~s}^{-1}$. To derive the mean, we excluded clusters with absolute differences larger than $500 \mathrm{~km} \mathrm{~s}^{-1}$. In this case median and mean agree. The question remains whether this is a significant deviation. The absolute scale of the correlation velocities is based on the heliocentric velocity of NGC 1396, for which we give an error of $8 \mathrm{~km} \mathrm{~s}^{-1}$. Taking this into account, the deviation from zero of the differences is $-6 \pm 8 \mathrm{~km} \mathrm{~s}^{-1}$ and hence well inside the given uncertainty.

For mask No. 80 the spectra extraction and subsequent velocity determination has been done independently by two of us (T. R. and B. D.), and the differences for the line measurements are plotted in Figure 5. The standard deviation between the two measurements is $\sigma=32 \mathrm{~km} \mathrm{~s}^{-1}$, while we 

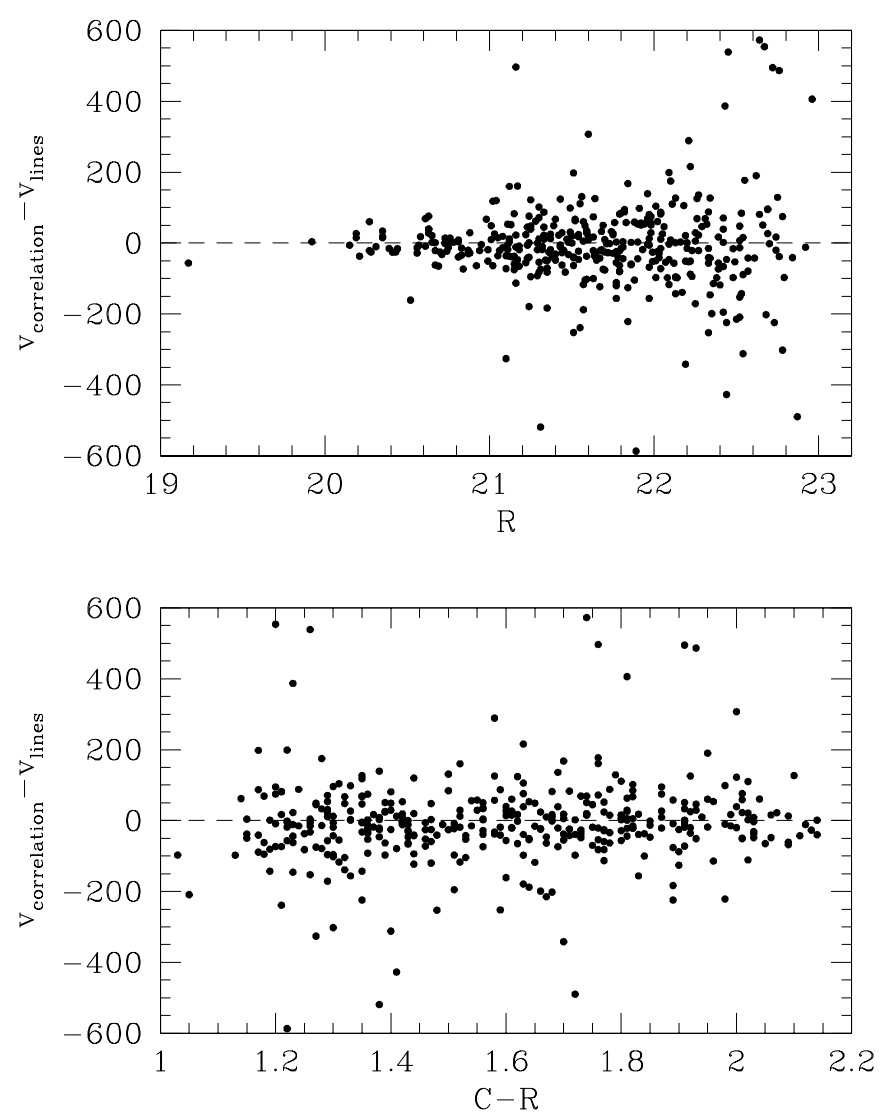

FIG. 4.-Difference between the velocities determined via line measurements and the correlation technique (top) vs. the brightness of the objects. It is expected and visible that for fainter objects the differences increase. Bottom: Differences plotted vs. their colors. No correlation with color can be seen.

expect $37 \mathrm{~km} \mathrm{~s}^{-1}$ from the line measurement errors. The scatter reflects the uncertainty resulting from independent treatment (tracing, aperture definition, wavelength calibration, line identification) of the data.

The best estimation of the uncertainties is a comparison of measurements that are obtained on different masks, which has been done for 31 point sources ( 29 clusters and two stars). The differences between the velocities are plotted in Figure 6, in dependence on brightness and color. The scatter of the

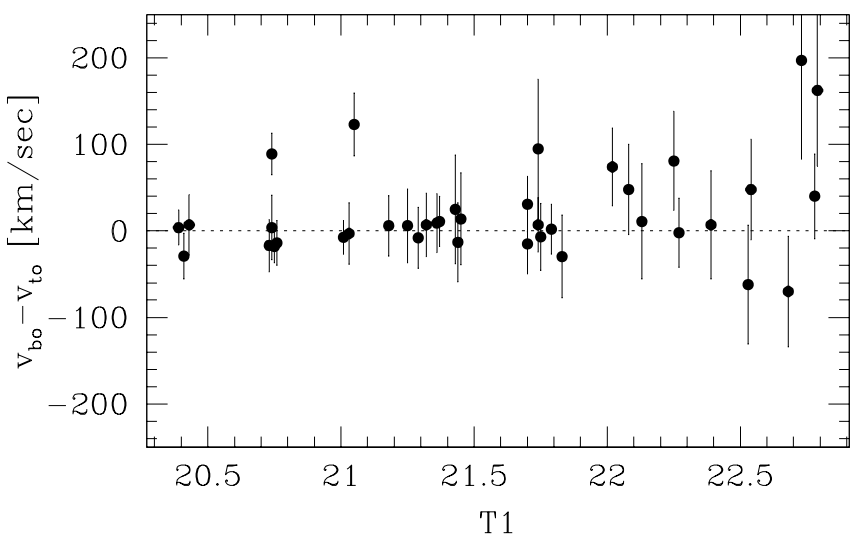

FIG. 5.-Differences of the velocities determined on mask 80 by line measurements by different authors, TR $\left(v_{\mathrm{to}}\right)$ and $\mathrm{BD}\left(v_{\mathrm{bo}}\right)$. The scatter reflects the uncertainty resulting from independent treatment (tracing, wavelength calibration, line identification) of the data.
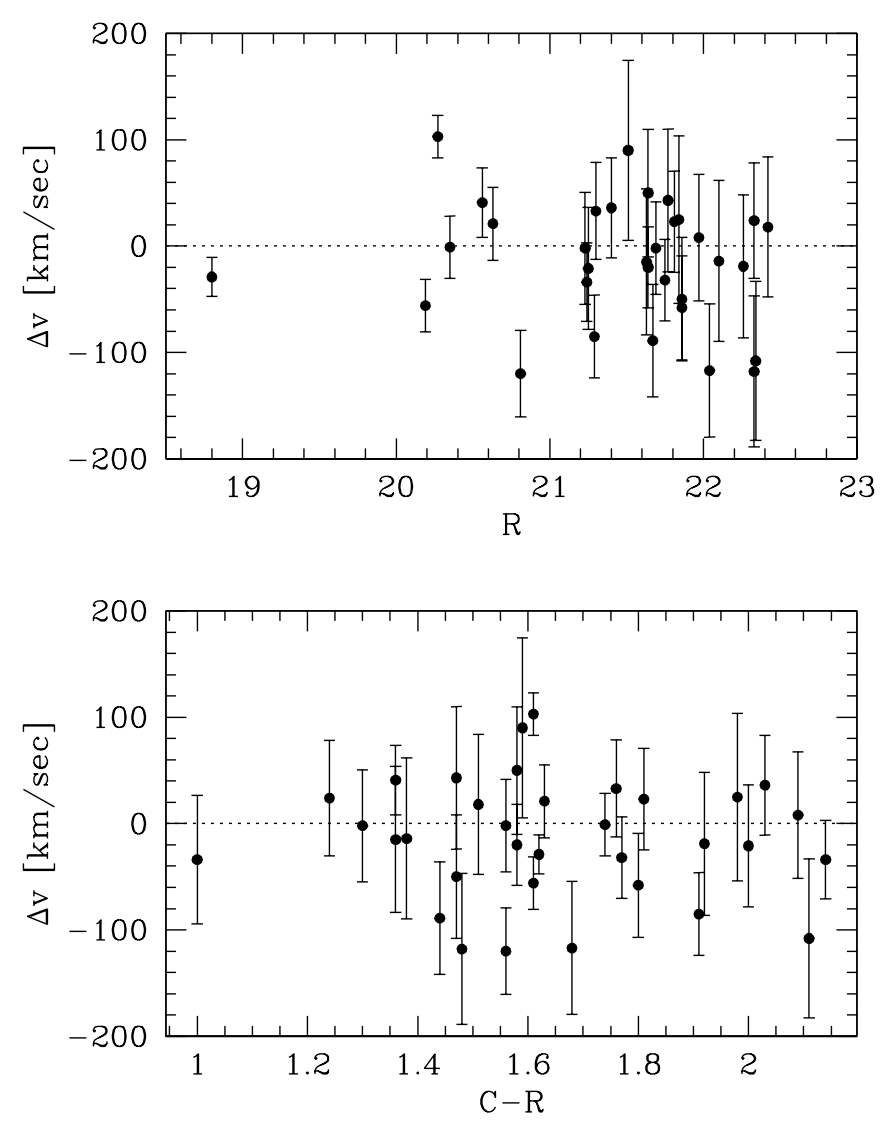

FIG. 6.-Differences of the correlation velocities for common objects on different masks. No systematic difference can be seen. The scatter is compatible with the mean uncertainties of the velocities (see $\S 5.2$ ).

velocities measured via correlation is $57 \mathrm{~km} \mathrm{~s}^{-1}$ and in very good agreement with the expectation from the individual uncertainties $\left(52 \mathrm{~km} \mathrm{~s}^{-1}\right)$, which shows that the given uncertainties are correct. The scatter of the velocities based on direct line measurements is $79 \mathrm{~km} \mathrm{~s}^{-1}$ and much larger than expected from the individual errors $\left(40 \mathrm{~km} \mathrm{~s}^{-1}\right)$. This indicates that the errors of the line measurements are dominated by systematic errors and are not of a statistical nature. The reason for the systematic errors is most probably line misidentification in noisy spectra. Line misidentification in noisy spectra also accounts for small velocity uncertainties at relatively faint clusters.

Summarizing, we suggest using the correlation velocities for any purpose; however, we provide the line measurements for a consistency check. For this reason, we do not attempt to derive a more refined uncertainty estimation for the line velocities.

\subsection{The Final Sample}

The whole data set is tabulated in Table 3 . The first column identifies the cluster: we used an identifier consisting of two two-digit numbers, the first indicating the mask and the second the aperture number on the mask that was assigned during the extraction process. Columns (2) and (3) are right ascension and declination of the clusters (J2000.0). The positions are based on the USNO-2.0 catalog ${ }^{12}$ (Urban et al. 1998). The color and magnitude information in columns (4) and (5) are taken from Dirsch et al. (2003). The uncertainties

\footnotetext{
${ }^{12}$ See http://tdc-www.harvard.edu/software/catalogs/ua2.html.
} 
TABLE 3

Globular Clusters for Which Velocities Have Been Determined with Lines and/or Cross Correlation ${ }^{a}$

\begin{tabular}{|c|c|c|c|c|c|c|c|}
\hline $\begin{array}{c}\text { Identifier } \\
\text { (1) }\end{array}$ & $\begin{array}{c}\text { R.A. (J2000.0) } \\
(2)\end{array}$ & $\begin{array}{c}\text { Decl. (J2000.0) } \\
\text { (3) }\end{array}$ & $\begin{array}{l}C-R \\
\text { (4) }\end{array}$ & $\begin{array}{c}R \\
(5)\end{array}$ & $\begin{array}{c}v_{\mathrm{c}} \\
\left(\mathrm{km} \mathrm{s}^{-1}\right) \\
(6)\end{array}$ & $\begin{array}{c}v_{l} \\
\left(\mathrm{~km} \mathrm{~s}^{-1}\right) \\
(7)\end{array}$ & $\begin{array}{c}\text { Comments } \\
\text { (8) }\end{array}$ \\
\hline 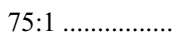 & 33855.58 & -352652.4 & $1.17 \pm 0.03$ & $21.33 \pm 0.01$ & $1481 \pm 52$ & $1394 \pm 31$ & \\
\hline 75:8 ................... & 33850.62 & -352632.3 & $1.31 \pm 0.05$ & $22.09 \pm 0.03$ & $1137 \pm 65$ & $1254 \pm 29$ & \\
\hline 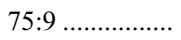 & 33901.54 & -352630.1 & $1.38 \pm 0.03$ & $21.31 \pm 0.02$ & $1419 \pm 47$ & $1938 \pm 22$ & \\
\hline 75:24 ............... & 33901.50 & -352530.6 & $1.51 \pm 0.09$ & $22.48 \pm 0.04$ & $1303 \pm 59$ & $1400 \pm 54$ & \\
\hline $75: 25 \ldots \ldots \ldots \ldots$ & 33853.08 & -352527.3 & $1.30 \pm 0.08$ & $22.69 \pm 0.04$ & $1804 \pm 57$ & $1708 \pm 41$ & \\
\hline 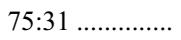 & 33858.86 & -352505.6 & $1.38 \pm 0.03$ & $22.10 \pm 0.02$ & $1572 \pm 51$ & $1563 \pm 31$ & $78: 11:: 1579 \pm 44$ \\
\hline 75:33 ............... & 33855.86 & -352459.6 & $1.24 \pm 0.06$ & $22.33 \pm 0.04$ & $1616 \pm 38$ & $1528 \pm 32$ & $78: 22:: 1604 \pm 39$ \\
\hline 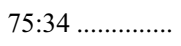 & 33849.74 & -352457.6 & $2.09 \pm 0.08$ & $21.97 \pm 0.04$ & $1473 \pm 42$ & $1460 \pm 25$ & $78: 43:: 1469 \pm 42$ \\
\hline $75: 35 \ldots \ldots \ldots \ldots$ & 33848.23 & -352454.2 & $1.48 \pm 0.06$ & $22.33 \pm 0.03$ & $1154 \pm 38$ & $1407 \pm 55$ & $78: 49:: 1213 \pm 49$ \\
\hline 75:36 ................. & 33857.38 & -352450.1 & $1.61 \pm 0.02$ & $20.19 \pm 0.01$ & $893 \pm 17$ & $866 \pm 15$ & $78: 15:: 921 \pm 18$ \\
\hline $75: 37 \ldots \ldots \ldots \ldots \ldots$ & 33850.74 & -352448.1 & $1.35 \pm 0.06$ & $22.29 \pm 0.03$ & $1293 \pm 47$ & $1246 \pm 37$ & \\
\hline 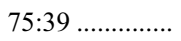 & 33854.48 & -352443.1 & $1.72 \pm 0.13$ & $22.87 \pm 0.06$ & $1406 \pm 36$ & $1896 \pm 40$ & \\
\hline 75:43 ................ & 33850.59 & -352429.1 & $1.58 \pm 0.04$ & $21.64 \pm 0.02$ & $1662 \pm 26$ & $1694 \pm 40$ & $76: 47,78: 40:: 1652 \pm 36$ \\
\hline 75:44 ................... & 33850.69 & -352426.9 & $2.10 \pm 0.11$ & $22.34 \pm 0.02$ & $1371 \pm 34$ & $1244 \pm 73$ & \\
\hline 75:46 .................. & 33851.07 & -352420.6 & $2.03 \pm 0.05$ & $21.40 \pm 0.02$ & $1301 \pm 30$ & $1349 \pm 29$ & $78: 38:: 1283 \pm 33$ \\
\hline 75:51 ............... & 33901.44 & -352358.0 & & & $1715 \pm 93$ & $1875 \pm 29$ & \\
\hline 75:56 .................. & 33856.37 & -352331.6 & $1.53 \pm 0.07$ & $22.21 \pm 0.04$ & $725 \pm 50$ & $778 \pm 29$ & \\
\hline $75: 68 \ldots \ldots \ldots \ldots \ldots$ & 33849.33 & -352259.0 & $1.36 \pm 0.04$ & $21.29 \pm 0.03$ & $1335 \pm 55$ & $1427 \pm 19$ & \\
\hline 75:71 .............. & 33848.90 & -352250.1 & $1.47 \pm 0.05$ & $21.77 \pm 0.02$ & $1674 \pm 41$ & $1794 \pm 28$ & $76: 77:: 1653 \pm 47$ \\
\hline $75: 73 \ldots \ldots \ldots \ldots . . .$. & 33855.08 & -352242.1 & $2.01 \pm 0.15$ & $22.84 \pm 0.06$ & $1422 \pm 61$ & $\ldots$ & \\
\hline 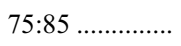 & 33850.37 & -352207.8 & $1.78 \pm 0.03$ & $20.83 \pm 0.02$ & $1609 \pm 24$ & $1643 \pm 23$ & \\
\hline $75: 92 \ldots \ldots \ldots \ldots \ldots$ & 33849.13 & $\begin{array}{lll}-35 & 21 & 42.2\end{array}$ & $1.74 \pm 0.02$ & $20.35 \pm 0.01$ & $889 \pm 18$ & $870 \pm 15$ & $76: 97:: 890 \pm 21$ \\
\hline $75: 93 \ldots \ldots \ldots \ldots \ldots$ & 33854.22 & -352137.9 & $1.74 \pm 0.14$ & $22.85 \pm 0.04$ & $1261 \pm 81$ & $\ldots$ & \\
\hline 75:95 ................. & 33859.37 & -352129.6 & $1.35 \pm 0.03$ & $21.40 \pm 0.02$ & $760 \pm 44$ & $693 \pm 21$ & \\
\hline 75:99 ................ & 33857.76 & -352112.2 & $1.29 \pm 0.03$ & $21.49 \pm 0.02$ & $1723 \pm 43$ & $1692 \pm 15$ & \\
\hline 75:106 ................ & 33900.80 & -352049.6 & $1.58 \pm 0.04$ & $21.92 \pm 0.02$ & $1687 \pm 47$ & $1630 \pm 28$ & \\
\hline 75:111 .............. & 33859.02 & -352032.7 & $1.19 \pm 0.04$ & $22.13 \pm 0.02$ & $1640 \pm 93$ & $1639 \pm 41$ & \\
\hline $76: 2 \ldots \ldots \ldots \ldots \ldots$ & 33840.50 & -352646.2 & $1.70 \pm 0.06$ & $21.84 \pm 0.02$ & $1703 \pm 57$ & $1535 \pm 34$ & \\
\hline 76:4 …................. & 33852.71 & -352640.5 & $1.59 \pm 0.04$ & $22.00 \pm 0.02$ & $1175 \pm 44$ & & \\
\hline $76: 6 \ldots \ldots \ldots \ldots \ldots$ & 33842.41 & -352630.9 & $1.36 \pm 0.03$ & $21.88 \pm 0.02$ & $1654 \pm 63$ & $\ldots$ & \\
\hline $76: 10 \ldots \ldots \ldots \ldots \ldots$ & 33840.75 & -352621.0 & $1.28 \pm 0.06$ & $22.10 \pm 0.02$ & $1817 \pm 48$ & $1642 \pm 30$ & \\
\hline $76: 11 \ldots \ldots \ldots \ldots \ldots$ & 33842.09 & -352618.6 & $1.75 \pm 0.04$ & $21.33 \pm 0.02$ & $1369 \pm 37$ & $1324 \pm 35$ & \\
\hline $76: 13 \ldots \ldots \ldots \ldots . . . .$. & 33842.58 & -352612.7 & $1.76 \pm 0.04$ & $21.30 \pm 0.02$ & $1141 \pm 22$ & $1069 \pm 26$ & $78: 70:: 1125 \pm 31$ \\
\hline $76: 16 \ldots \ldots \ldots \ldots$ & 33847.51 & -352606.1 & $1.81 \pm 0.07$ & $22.05 \pm 0.04$ & $1656 \pm 41$ & $1630 \pm 20$ & \\
\hline $76: 18 \ldots \ldots \ldots \ldots$ & 33841.85 & -352600.7 & $2.05 \pm 0.03$ & $20.69 \pm 0.02$ & $1803 \pm 33$ & $1868 \pm 27$ & \\
\hline $76: 20 \ldots \ldots \ldots \ldots$ & 33846.41 & -352553.9 & $1.33 \pm 0.04$ & $21.91 \pm 0.02$ & $1282 \pm 31$ & $1184 \pm 37$ & \\
\hline 76:24 ................ & 33843.43 & $-35 \quad 2540.2$ & $1.29 \pm 0.04$ & $21.47 \pm 0.03$ & $1977 \pm 43$ & $1966 \pm 34$ & \\
\hline $76: 28 \ldots \ldots \ldots \ldots$ & 33845.88 & -352528.2 & $1.66 \pm 0.09$ & $22.35 \pm 0.03$ & $1518 \pm 39$ & $1717 \pm 43$ & \\
\hline $76: 30 \ldots \ldots \ldots \ldots$ & 33841.25 & -352523.5 & $1.74 \pm 0.10$ & $22.64 \pm 0.03$ & $1680 \pm 66$ & $1107 \pm 33$ & \\
\hline $76: 36 \ldots \ldots \ldots \ldots$ & 33844.01 & $\begin{array}{lll}-35 & 25 & 04.2\end{array}$ & $1.70 \pm 0.04$ & $21.38 \pm 0.02$ & $1401 \pm 29$ & $1439 \pm 26$ & \\
\hline $76: 38 \ldots \ldots \ldots \ldots . . .$. & 33838.64 & -352459.0 & $1.36 \pm 0.03$ & $21.63 \pm 0.02$ & $1023 \pm 50$ & $949 \pm 38$ & $77: 33:: 1031 \pm 49$ \\
\hline $76: 40 \ldots \ldots \ldots \ldots$ & 33844.21 & -352453.7 & $1.29 \pm 0.06$ & $22.42 \pm 0.04$ & $1194 \pm 37$ & $1123 \pm 36$ & \\
\hline 76:41 ................ & 33847.34 & -352450.3 & $1.76 \pm 0.04$ & $21.17 \pm 0.01$ & $1031 \pm 34$ & $870 \pm 33$ & \\
\hline 76:42 …............. & 33847.89 & -352447.6 & $1.59 \pm 0.12$ & $22.55 \pm 0.03$ & $2417 \pm 55$ & $\ldots$ & \\
\hline $76: 43 \ldots \ldots \ldots \ldots . . . .$. & 33841.82 & -352443.1 & $2.14 \pm 0.06$ & $21.24 \pm 0.02$ & $1502 \pm 28$ & $1542 \pm 27$ & $78: 73:: 1519 \pm 50$ \\
\hline $76: 46 \ldots \ldots \ldots \ldots \ldots$ & 33840.84 & -352432.7 & $1.98 \pm 0.02$ & $21.49 \pm 0.01$ & $1349 \pm 32$ & $1250 \pm 39$ & \\
\hline $76: 47 \ldots \ldots \ldots \ldots$ & 33850.59 & -352429.0 & $1.58 \pm 0.04$ & $21.64 \pm 0.02$ & $1682 \pm 28$ & $1556 \pm 36$ & $75: 43,78: 40$ \\
\hline 76:51 …............. & 33843.27 & -352414.5 & $1.91 \pm 0.17$ & $22.72 \pm 0.03$ & $496 \pm 43$ & $\ldots$ & \\
\hline 76:54 ................. & 33843.57 & $-35 \quad 2404.1$ & $1.61 \pm 0.09$ & $21.02 \pm 0.09$ & $1514 \pm 34$ & & \\
\hline $76: 59 \ldots \ldots \ldots \ldots . . . .$. & 33846.90 & -352348.4 & $\ldots$ & $\ldots$ & $1652 \pm 32$ & $1679 \pm 20$ & \\
\hline 76:61 …............. & 33845.65 & -352342.3 & $\ldots$ & $\ldots$ & $1146 \pm 44$ & $975 \pm 46$ & \\
\hline 76:63 ................. & 33849.85 & -352335.8 & $1.98 \pm 0.08$ & $20.31 \pm 0.04$ & $933 \pm 29$ & $943 \pm 21$ & \\
\hline 76:65 .................. & 33844.54 & -352330.1 & $1.40 \pm 0.09$ & $22.54 \pm 0.02$ & $1547 \pm 99$ & $1859 \pm 33$ & \\
\hline $76: 67 \ldots \ldots \ldots \ldots \ldots$ & 33843.04 & -352321.5 & $2.11 \pm 0.11$ & $22.34 \pm 0.04$ & $1565 \pm 29$ & $1608 \pm 39$ & $77: 64:: 1619 \pm 49$ \\
\hline $76: 73 \ldots \ldots \ldots \ldots$. & 33841.80 & -352304.9 & $2.00 \pm 0.07$ & $21.25 \pm 0.03$ & $1466 \pm 52$ & $1344 \pm 46$ & $77: 70:: 1477 \pm 38$ \\
\hline $76: 76 \ldots \ldots \ldots \ldots \ldots$ & 33840.40 & -352253.5 & $1.68 \pm 0.06$ & $22.04 \pm 0.02$ & $1923 \pm 39$ & $1912 \pm 30$ & $77: 74:: 1982 \pm 44$ \\
\hline 76:77 …................ & 33848.90 & -352250.0 & $1.47 \pm 0.05$ & $21.77 \pm 0.02$ & $1631 \pm 53$ & $1628 \pm 35$ & $75: 71$ \\
\hline $76: 80 \ldots \ldots \ldots \ldots \ldots$ & 33841.01 & $\begin{array}{lll}-35 & 22 & 42.2\end{array}$ & $1.69 \pm 0.03$ & $20.67 \pm 0.01$ & $1654 \pm 19$ & $1675 \pm 20$ & \\
\hline 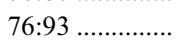 & 33843.39 & -352159.1 & $1.92 \pm 0.05$ & $21.37 \pm 0.02$ & $1395 \pm 29$ & $1370 \pm 19$ & \\
\hline $76: 97 \ldots \ldots \ldots \ldots \ldots$ & 33849.14 & -352142.2 & $1.74 \pm 0.02$ & $20.35 \pm 0.01$ & $890 \pm 23$ & $875 \pm 19$ & $75: 92$ \\
\hline 76:98 .................. & 33840.30 & -352139.9 & $2.01 \pm 0.04$ & $21.35 \pm 0.02$ & $1549 \pm 30$ & $1599 \pm 36$ & \\
\hline 76:101 .............. & 33840.24 & -352133.3 & $1.65 \pm 0.03$ & $20.86 \pm 0.02$ & $1822 \pm 31$ & & \\
\hline $76: 104 \ldots \ldots \ldots \ldots$ & 33848.91 & -352122.4 & $2.05 \pm 0.03$ & $20.98 \pm 0.02$ & $1637 \pm 34$ & & \\
\hline
\end{tabular}


TABLE 3-Continued

\begin{tabular}{|c|c|c|c|c|c|c|c|}
\hline $\begin{array}{c}\text { Identifier } \\
\text { (1) }\end{array}$ & $\begin{array}{l}\text { R.A. (J2000.0) } \\
\text { (2) }\end{array}$ & $\begin{array}{l}\text { Decl. (J2000.0) } \\
\text { (3) }\end{array}$ & $\begin{array}{l}C-R \\
(4)\end{array}$ & $\begin{array}{c}R \\
(5)\end{array}$ & $\begin{array}{c}v_{\mathrm{c}} \\
\left(\mathrm{km} \mathrm{s}^{-1}\right) \\
(6)\end{array}$ & $\begin{array}{c}v_{l} \\
\left(\mathrm{~km} \mathrm{~s}^{-1}\right) \\
(7)\end{array}$ & $\begin{array}{c}\text { Comments } \\
\text { (8) }\end{array}$ \\
\hline 76:106 ........... & 33842.35 & $\begin{array}{lll}-35 & 21 & 17.1\end{array}$ & $1.80 \pm 0.05$ & $21.86 \pm 0.03$ & $1833 \pm 37$ & $\ldots$ & $77: 105:: 1862 \pm 35$ \\
\hline 76:108 .............. & 33840.19 & -352109.2 & $1.47 \pm 0.05$ & $21.86 \pm 0.02$ & $1238 \pm 39$ & $\ldots$ & $77: 107:: 1263 \pm 41$ \\
\hline $76: 112 \ldots \ldots \ldots \ldots$ & 33846.98 & -353050.7 & $1.34 \pm 0.04$ & $21.64 \pm 0.03$ & $1431 \pm 98$ & $\ldots$ & \\
\hline $76: 123 \ldots \ldots \ldots \ldots$ & 33840.51 & -352006.1 & $1.48 \pm 0.10$ & $22.56 \pm 0.03$ & $1280 \pm 57$ & $\ldots$ & \\
\hline $77: 2 \ldots \ldots \ldots \ldots . .$. & 33838.22 & -352645.2 & & & $1815 \pm 34$ & $\ldots$ & \\
\hline 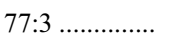 & 33841.63 & -352642.4 & $1.21 \pm 0.09$ & $22.74 \pm 0.04$ & $1783 \pm 28$ & $1766 \pm 25$ & \\
\hline $77: 4 \ldots \ldots \ldots \ldots . . . .$. & 33838.88 & -352638.6 & $1.27 \pm 0.09$ & $22.88 \pm 0.04$ & $1303 \pm 43$ & $\ldots$ & \\
\hline 77:12 ............. & 33838.12 & -352614.9 & $1.93 \pm 0.13$ & $22.76 \pm 0.05$ & $2206 \pm 53$ & $1719 \pm 45$ & \\
\hline 77:14 „............ & 33840.74 & -352608.2 & $1.21 \pm 0.04$ & $21.79 \pm 0.03$ & $1222 \pm 66$ & $1140 \pm 31$ & \\
\hline 77:16 ............ & 33838.52 & -352601.0 & $\ldots$ & $\ldots$ & $1415 \pm 53$ & $\ldots$ & \\
\hline 77:18 ............ & 33842.21 & -352555.1 & $1.52 \pm 0.04$ & $21.57 \pm 0.02$ & $1805 \pm 42$ & $1922 \pm 35$ & \\
\hline 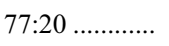 & 33834.94 & $-35 \quad 2547.2$ & $1.44 \pm 0.05$ & $22.03 \pm 0.04$ & $1282 \pm 42$ & $1323 \pm 40$ & \\
\hline 77:21 ............. & 33838.76 & $\begin{array}{lll}-35 & 25 & 42.9\end{array}$ & $1.85 \pm 0.03$ & $21.08 \pm 0.02$ & $1705 \pm 18$ & $1718 \pm 22$ & \\
\hline 77:23 .............. & 33834.59 & -352536.8 & $1.81 \pm 0.05$ & $21.52 \pm 0.03$ & $1242 \pm 28$ & $1179 \pm 33$ & \\
\hline $77: 24 \ldots \ldots \ldots \ldots$ & 33835.79 & $-35 \quad 2534.2$ & $1.30 \pm 0.03$ & $21.23 \pm 0.03$ & $1353 \pm 29$ & $1396 \pm 22$ & $78: 94:: 1354 \pm 37$ \\
\hline $77: 25 \ldots \ldots \ldots \ldots$ & 33832.61 & $-35 \quad 2529.8$ & $1.89 \pm 0.08$ & $21.70 \pm 0.04$ & $1686 \pm 37$ & $1762 \pm 22$ & \\
\hline 77:31 ............... & 33838.79 & $\begin{array}{lll}-35 & 25 & 04.3\end{array}$ & $1.33 \pm 0.04$ & $22.16 \pm 0.03$ & $1253 \pm 40$ & $1250 \pm 24$ & \\
\hline 77:33 …........... & 33838.63 & -352459.0 & $1.36 \pm 0.03$ & $21.63 \pm 0.02$ & $1038 \pm 47$ & $1059 \pm 27$ & $76: 38$ \\
\hline $77: 35 \ldots \ldots \ldots \ldots$ & 33831.61 & -352453.8 & $1.80 \pm 0.07$ & $22.03 \pm 0.04$ & $1581 \pm 41$ & $1610 \pm 26$ & \\
\hline 77:39 ................ & 33838.25 & -352443.3 & $1.40 \pm 0.03$ & $21.44 \pm 0.02$ & $1859 \pm 64$ & $1830 \pm 33$ & \\
\hline 77:40 ............. & 33830.80 & -352439.0 & $\ldots$ & $\ldots$ & $1334 \pm 13$ & $\ldots$ & \\
\hline 77:42 …......... & 33834.27 & -352435.6 & $1.85 \pm 0.16$ & $22.99 \pm 0.05$ & $1288 \pm 58$ & $\ldots$ & \\
\hline 77:43 …........... & 33839.08 & -352432.7 & $1.44 \pm 0.09$ & $22.81 \pm 0.04$ & $1391 \pm 33$ & $\ldots$ & \\
\hline 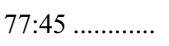 & 33838.63 & -352427.1 & $1.27 \pm 0.03$ & $21.15 \pm 0.02$ & $1614 \pm 39$ & $1689 \pm 18$ & \\
\hline 77:47 ............. & 33836.81 & -352419.3 & $1.26 \pm 0.06$ & $22.04 \pm 0.03$ & $1443 \pm 43$ & $1458 \pm 27$ & \\
\hline 77:63 .............. & 33833.65 & -352322.7 & $\ldots$ & $\ldots$ & $1156 \pm 57$ & $\ldots$ & \\
\hline 77:64 ............... & 33843.04 & -352321.6 & $2.11 \pm 0.11$ & $22.34 \pm 0.04$ & $1673 \pm 69$ & $\ldots$ & $76: 67$ \\
\hline 77:70 ..................... & 33841.80 & -352304.9 & $2.00 \pm 0.07$ & $21.25 \pm 0.03$ & $1487 \pm 24$ & $1448 \pm 28$ & $76: 73$ \\
\hline 77:74 …........... & 33840.40 & -352253.4 & $1.68 \pm 0.06$ & $22.04 \pm 0.02$ & $2040 \pm 49$ & $1958 \pm 25$ & $76: 76$ \\
\hline 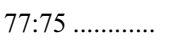 & 33835.89 & -352249.6 & $1.91 \pm 0.10$ & $22.66 \pm 0.04$ & $1535 \pm 28$ & $1484 \pm 31$ & \\
\hline 77:77 .................. & 33841.09 & $-35 \quad 2240.5$ & $\ldots$ & $\ldots$ & $1690 \pm 14$ & $\ldots$ & \\
\hline 77:80 ............... & 33837.81 & -352233.8 & $1.67 \pm 0.10$ & $22.58 \pm 0.03$ & $1258 \pm 21$ & $\ldots$ & \\
\hline $77: 84 \ldots \ldots \ldots \ldots$ & 33833.25 & $-35 \quad 22 \quad 18.0$ & $1.34 \pm 0.05$ & $22.19 \pm 0.03$ & $1302 \pm 49$ & $\ldots$ & \\
\hline $77: 86 \ldots \ldots \ldots . .$. & 33833.46 & $\begin{array}{lll}-35 & 22 & 12.4\end{array}$ & $1.22 \pm 0.03$ & $21.89 \pm 0.02$ & $808 \pm 46$ & $1395 \pm 33$ & \\
\hline 77:92 …............ & 33833.90 & -352154.8 & $1.82 \pm 0.05$ & $21.52 \pm 0.03$ & $1293 \pm 29$ & $1226 \pm 30$ & \\
\hline 77:105 ........... & 33842.26 & $-3521 \quad 17.1$ & $1.80 \pm 0.05$ & $21.86 \pm 0.03$ & $1891 \pm 32$ & $1889 \pm 23$ & $76: 106$ \\
\hline 77:106 ............... & 33837.74 & $-3521 \quad 13.0$ & $1.38 \pm 0.07$ & $22.44 \pm 0.04$ & $856 \pm 51$ & $902 \pm 36$ & \\
\hline 77:107 ........... & 33840.20 & $\begin{array}{llll}-35 & 21 & 09.2\end{array}$ & $1.47 \pm 0.05$ & $21.86 \pm 0.02$ & $1288 \pm 43$ & $1347 \pm 33$ & $76: 108$ \\
\hline $77: 112 \ldots \ldots \ldots \ldots$ & 33837.02 & $-35 \quad 2052.2$ & $1.72 \pm 0.05$ & $21.78 \pm 0.03$ & $1563 \pm 29$ & $1602 \pm 26$ & \\
\hline 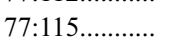 & 33838.74 & -352039.9 & $1.50 \pm 0.23$ & $22.53 \pm 0.04$ & $1471 \pm 57$ & $1387 \pm 44$ & \\
\hline 77:127 ........... & 33835.60 & -352005.9 & $1.27 \pm 0.03$ & $21.23 \pm 0.02$ & $1546 \pm 27$ & $1501 \pm 30$ & \\
\hline 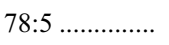 & 33900.43 & -352609.7 & $1.39 \pm 0.05$ & $21.79 \pm 0.03$ & $1813 \pm 40$ & $1876 \pm 40$ & \\
\hline 78:11 ................. & 33858.86 & -352505.7 & $1.27 \pm 0.03$ & $21.10 \pm 0.02$ & $1586 \pm 56$ & $1912 \pm 35$ & $75: 31$ \\
\hline $78: 12 \ldots \ldots \ldots \ldots$ & 33858.49 & -352628.1 & $1.26 \pm 0.03$ & $19.92 \pm 0.02$ & $1048 \pm 18$ & $1044 \pm 21$ & \\
\hline 78:13 .............. & 33858.10 & -352621.9 & $1.66 \pm 0.05$ & $21.77 \pm 0.02$ & $1337 \pm 40$ & $1361 \pm 34$ & \\
\hline $78: 15 \ldots \ldots \ldots \ldots$ & 33857.37 & -352450.1 & $1.61 \pm 0.02$ & $20.19 \pm 0.01$ & $949 \pm 18$ & $934 \pm 22$ & $75: 36$ \\
\hline 78:22 …............ & 33855.87 & -352459.6 & $1.24 \pm 0.06$ & $22.33 \pm 0.04$ & $1592 \pm 39$ & $1607 \pm 38$ & $75: 33$ \\
\hline 78:28 .............. & 33854.20 & -352436.2 & $1.72 \pm 0.09$ & $22.87 \pm 0.04$ & $811 \pm 48$ & & \\
\hline 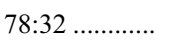 & 33853.08 & $\begin{array}{lll}-35 & 25 & 27.3\end{array}$ & $1.33 \pm 0.08$ & $22.69 \pm 0.04$ & $873 \pm 48$ & $846 \pm 35$ & \\
\hline 78:35 .............. & 33851.91 & -352430.8 & $1.30 \pm 0.09$ & $22.78 \pm 0.05$ & $1046 \pm 137$ & $1348 \pm 34$ & \\
\hline 78:38 ….................... & 33851.07 & -352420.8 & $2.03 \pm 0.05$ & $21.40 \pm 0.02$ & $1265 \pm 36$ & $1296 \pm 23$ & $75: 46$ \\
\hline 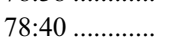 & 33850.59 & -352429.0 & $1.58 \pm 0.04$ & $21.64 \pm 0.02$ & $1612 \pm 54$ & $1650 \pm 28$ & $75: 43,76: 47$ \\
\hline 78:43 …........... & 33849.75 & -352457.7 & $2.09 \pm 0.08$ & $21.97 \pm 0.04$ & $1465 \pm 42$ & $1533 \pm 36$ & $75: 34$ \\
\hline $78: 48 \ldots \ldots \ldots \ldots . . .$. & 33848.47 & -352505.0 & $1.93 \pm 0.07$ & $22.04 \pm 0.02$ & $2008 \pm 43$ & $\ldots$ & \\
\hline 78:49 .................. & 33848.23 & -352454.0 & $1.48 \pm 0.06$ & $22.33 \pm 0.03$ & $1272 \pm 60$ & $1313 \pm 35$ & $75: 35$ \\
\hline 78:58 ............... & 33845.91 & -352441.6 & $2.05 \pm 0.12$ & $22.91 \pm 0.05$ & $1158 \pm 64$ & $\ldots$ & \\
\hline 78:61 .............. & 33844.79 & $\begin{array}{llll}-35 & 25 & 22.3\end{array}$ & $1.89 \pm 0.08$ & $22.44 \pm 0.03$ & $843 \pm 68$ & $1067 \pm 41$ & \\
\hline 78:66 …............ & 33843.53 & -352603.8 & $1.70 \pm 0.07$ & $22.19 \pm 0.04$ & $1381 \pm 40$ & $1723 \pm 35$ & \\
\hline 78:70 ............... & 33842.56 & -352612.7 & $1.76 \pm 0.04$ & $21.30 \pm 0.02$ & $1108 \pm 40$ & $1162 \pm 30$ & $76: 13$ \\
\hline 78:71 .............. & 33842.32 & -352611.9 & $1.53 \pm 0.08$ & $22.57 \pm 0.04$ & $1327 \pm 35$ & $1369 \pm 28$ & \\
\hline 78:73 .............. & 33841.82 & -352443.0 & $2.14 \pm 0.06$ & $21.24 \pm 0.02$ & $1536 \pm 24$ & $1535 \pm 22$ & $76: 43$ \\
\hline 78:82 …........... & 33839.42 & -352541.9 & $1.77 \pm 0.03$ & $21.13 \pm 0.02$ & $1387 \pm 23$ & $1335 \pm 28$ & \\
\hline 78:84 .............. & 33838.79 & -352555.0 & $1.89 \pm 0.02$ & $20.61 \pm 0.01$ & $1021 \pm 37$ & $1030 \pm 21$ & \\
\hline 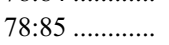 & 33838.47 & -352613.8 & $1.32 \pm 0.04$ & $21.88 \pm 0.02$ & $1419 \pm 56$ & $1523 \pm 38$ & \\
\hline
\end{tabular}


TABLE 3-Continued

\begin{tabular}{|c|c|c|c|c|c|c|c|}
\hline $\begin{array}{l}\text { Identifier } \\
\text { (1) }\end{array}$ & $\begin{array}{c}\text { R.A. (J2000.0) } \\
\text { (2) }\end{array}$ & $\begin{array}{c}\text { Decl. (J2000.0) } \\
\text { (3) }\end{array}$ & $\begin{array}{l}C-R \\
\text { (4) }\end{array}$ & $\begin{array}{c}R \\
(5)\end{array}$ & $\begin{array}{c}v_{\mathrm{c}} \\
\left(\mathrm{km} \mathrm{s}^{-1}\right) \\
(6)\end{array}$ & $\begin{array}{c}v_{l} \\
\left(\mathrm{~km} \mathrm{~s}^{-1}\right) \\
(7)\end{array}$ & $\begin{array}{c}\text { Comments } \\
\text { (8) }\end{array}$ \\
\hline 78:88 ................ & 33837.72 & -352601.7 & $1.67 \pm 0.05$ & $21.54 \pm 0.02$ & $1385 \pm 49$ & $1431 \pm 30$ & \\
\hline 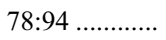 & 33835.79 & $\begin{array}{llll}-35 & 25 & 33.2\end{array}$ & $1.30 \pm 0.03$ & $21.23 \pm 0.03$ & $1355 \pm 44$ & $1361 \pm 23$ & $77: 24$ \\
\hline 78:95 …............ & 33835.50 & -352529.7 & $1.64 \pm 0.03$ & $20.99 \pm 0.02$ & $1302 \pm 31$ & $1353 \pm 26$ & \\
\hline 78:96 …........... & 33835.26 & $-35 \quad 2539.3$ & $1.56 \pm 0.03$ & $21.23 \pm 0.03$ & $1601 \pm 33$ & $\ldots$ & \\
\hline 78:98 ….......... & 33834.77 & $-35 \quad 2541.2$ & $1.33 \pm 0.04$ & $22.01 \pm 0.03$ & $1514 \pm 51$ & $\ldots$ & \\
\hline 78:102 ............ & 33833.26 & -352519.2 & $1.29 \pm 0.07$ & $22.25 \pm 0.04$ & $1040 \pm 43$ & $1211 \pm 30$ & \\
\hline 78:103 .............. & 33832.91 & -352353.7 & $1.20 \pm 0.06$ & $22.67 \pm 0.03$ & $555 \pm 37$ & & \\
\hline 78:106 .............. & 33832.12 & -352455.1 & $1.63 \pm 0.04$ & $21.14 \pm 0.02$ & $1288 \pm 26$ & $1327 \pm 30$ & \\
\hline 78:108 .................... & 33831.63 & -352433.4 & $1.82 \pm 0.05$ & $21.67 \pm 0.02$ & $1156 \pm 31$ & $1178 \pm 22$ & \\
\hline 78:109 ............ & 33831.34 & $-3524 \quad 17.3$ & $1.35 \pm 0.05$ & $21.96 \pm 0.03$ & $1720 \pm 64$ & $1651 \pm 28$ & \\
\hline 78:110............... & 33830.97 & -352431.9 & $1.83 \pm 0.07$ & $21.97 \pm 0.03$ & $1632 \pm 71$ & $1788 \pm 33$ & \\
\hline 78:113 .............. & 33830.17 & -352508.3 & $2.01 \pm 0.04$ & $21.24 \pm 0.02$ & $1065 \pm 39$ & $989 \pm 31$ & \\
\hline 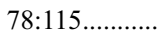 & 33829.53 & $\begin{array}{lll}-35 & 25 & 08.5\end{array}$ & $1.82 \pm 0.02$ & $20.74 \pm 0.01$ & $1358 \pm 34$ & $1353 \pm 22$ & \\
\hline 78:117............... & 33828.87 & -352500.9 & $1.69 \pm 0.06$ & $21.31 \pm 0.03$ & $1480 \pm 35$ & $1554 \pm 36$ & \\
\hline $80: 2 \ldots \ldots \ldots \ldots$ & 33822.70 & $\begin{array}{lll}-35 & 26 & 49.2\end{array}$ & $1.89 \pm 0.10$ & $21.83 \pm 0.06$ & $975 \pm 37$ & $917 \pm 27$ & \\
\hline $80: 4 \ldots \ldots \ldots \ldots . . .$. & 33820.03 & -352643.8 & $1.51 \pm 0.02$ & $20.74 \pm 0.02$ & $1471 \pm 21$ & $1494 \pm 23$ & \\
\hline $80: 6 \ldots \ldots \ldots \ldots . . .$. & 33819.10 & -352637.4 & $1.54 \pm 0.02$ & $20.39 \pm 0.01$ & $1524 \pm 18$ & $1539 \pm 14$ & \\
\hline $80: 7$................... & 33822.46 & -352632.8 & $1.50 \pm 0.02$ & $20.41 \pm 0.01$ & $1197 \pm 22$ & $1223 \pm 21$ & \\
\hline $80: 10 \ldots \ldots \ldots . .$. & 33824.68 & -352622.5 & $1.61 \pm 0.05$ & $21.45 \pm 0.02$ & $1206 \pm 43$ & $1226 \pm 23$ & \\
\hline $80: 12 \ldots \ldots \ldots . .$. & 33821.60 & -352615.5 & $1.41 \pm 0.04$ & $21.37 \pm 0.02$ & $1480 \pm 23$ & $1468 \pm 18$ & \\
\hline 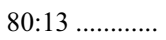 & 33820.64 & -352610.9 & $1.51 \pm 0.03$ & $21.18 \pm 0.02$ & $1799 \pm 23$ & $1807 \pm 22$ & \\
\hline $80: 15 \ldots \ldots \ldots . . . .$. & 33821.50 & -352605.1 & $1.76 \pm 0.10$ & $22.27 \pm 0.05$ & $1542 \pm 26$ & $1563 \pm 16$ & \\
\hline $80: 17 \ldots \ldots \ldots . . .$. & 33815.71 & -352600.4 & $1.77 \pm 0.04$ & $21.36 \pm 0.02$ & $1062 \pm 28$ & $1088 \pm 27$ & \\
\hline $80: 19 \ldots \ldots \ldots . . .$. & 33819.51 & -352552.4 & $1.90 \pm 0.02$ & $20.43 \pm 0.02$ & $1266 \pm 30$ & $1291 \pm 18$ & \\
\hline $80: 23 \ldots \ldots \ldots . .$. & 33827.39 & $\begin{array}{lll}-35 & 25 & 37.3\end{array}$ & $1.39 \pm 0.03$ & $21.03 \pm 0.02$ & $1421 \pm 24$ & $1395 \pm 21$ & \\
\hline $80: 24 \ldots \ldots \ldots . . .$. & 33826.67 & $\begin{array}{llll}-35 & 25 & 33.8\end{array}$ & $1.78 \pm 0.04$ & $21.05 \pm 0.02$ & $1457 \pm 24$ & $1442 \pm 19$ & \\
\hline $80: 26 \ldots \ldots \ldots \ldots$ & 33823.21 & -352529.6 & $2.13 \pm 0.06$ & $21.44 \pm 0.03$ & $1654 \pm 36$ & $1681 \pm 18$ & \\
\hline $80: 27 \ldots \ldots \ldots . .$. & 33826.27 & -352525.8 & $1.91 \pm 0.02$ & $20.74 \pm 0.01$ & $1260 \pm 33$ & $1259 \pm 22$ & \\
\hline $80: 28 \ldots \ldots \ldots . . .$. & 33826.46 & -352521.5 & $1.60 \pm 0.02$ & $20.76 \pm 0.01$ & $1753 \pm 18$ & $1739 \pm 17$ & \\
\hline $80: 30 \ldots \ldots \ldots \ldots$ & 33821.75 & -352514.9 & $1.91 \pm 0.05$ & $21.29 \pm 0.02$ & $1487 \pm 27$ & $1506 \pm 18$ & $82: 70:: 1530 \pm 28$ \\
\hline $80: 31 \ldots \ldots \ldots \ldots$ & 33816.86 & -352513.1 & $1.15 \pm 0.09$ & $22.76 \pm 0.04$ & $1390 \pm 45$ & $1428 \pm 32$ & \\
\hline $80: 33 \ldots \ldots \ldots . .$. & 33822.98 & -352503.7 & $1.81 \pm 0.06$ & $21.79 \pm 0.03$ & $1430 \pm 21$ & $1452 \pm 22$ & \\
\hline $80: 35 \ldots \ldots \ldots \ldots$ & 33818.27 & -352454.5 & $1.43 \pm 0.03$ & $20.75 \pm 0.02$ & $1530 \pm 17$ & $1542 \pm 16$ & \\
\hline $80: 40 \ldots \ldots \ldots \ldots$ & 33821.34 & -352435.7 & $1.67 \pm 0.03$ & $21.01 \pm 0.01$ & $881 \pm 18$ & $871 \pm 18$ & \\
\hline $80: 42 \ldots \ldots \ldots \ldots$ & 33823.24 & -352429.9 & $1.70 \pm 0.09$ & $22.25 \pm 0.04$ & $1252 \pm 51$ & $1246 \pm 38$ & \\
\hline $80: 44 \ldots \ldots \ldots \ldots$ & 33826.42 & -352425.1 & $1.68 \pm 0.03$ & $20.73 \pm 0.01$ & $1808 \pm 19$ & $1807 \pm 24$ & \\
\hline $80: 45 \ldots \ldots \ldots \ldots$ & 33822.86 & -352423.3 & $1.47 \pm 0.05$ & $21.70 \pm 0.04$ & $1568 \pm 28$ & $1593 \pm 20$ & \\
\hline $80: 46 \ldots \ldots \ldots \ldots$ & 33821.46 & -352420.4 & $1.20 \pm 0.04$ & $21.32 \pm 0.03$ & $1366 \pm 32$ & $1375 \pm 23$ & \\
\hline $80: 55 \ldots \ldots \ldots . . .$. & 33822.16 & -352343.1 & $\ldots$ & $\ldots$ & $979 \pm 34$ & $\ldots$ & \\
\hline $80: 56 \ldots \ldots \ldots . . .$. & 33819.80 & -352339.2 & $\ldots$ & $\ldots$ & $885 \pm 23$ & $887 \pm 19$ & \\
\hline 80:57 ................ & 33823.57 & -352332.6 & $1.67 \pm 0.02$ & $21.02 \pm 0.01$ & $1309 \pm 25$ & $1383 \pm 16$ & \\
\hline $80: 58 \ldots \ldots \ldots . . .$. & 33823.57 & $-3523 \quad 32.6$ & $1.67 \pm 0.02$ & $21.02 \pm 0.01$ & $1309 \pm 25$ & $1373 \pm 16$ & \\
\hline $80: 60 \ldots \ldots \ldots . . .$. & 33825.29 & -352325.3 & $1.94 \pm 0.04$ & $21.70 \pm 0.02$ & $1407 \pm 24$ & $1397 \pm 20$ & \\
\hline $80: 62 \ldots \ldots \ldots \ldots$ & 33821.54 & -352318.5 & $1.40 \pm 0.04$ & $22.64 \pm 0.03$ & $1568 \pm 48$ & $1487 \pm 36$ & \\
\hline $80: 63 \ldots \ldots \ldots \ldots$ & 33823.53 & -352315.8 & $1.87 \pm 0.05$ & $21.75 \pm 0.02$ & $1917 \pm 18$ & $1890 \pm 20$ & \\
\hline 80:71 ............... & 33817.70 & -352251.3 & $1.71 \pm 0.04$ & $22.02 \pm 0.03$ & $1515 \pm 28$ & $1539 \pm 24$ & \\
\hline 80:73 …....................... & 33825.52 & -352245.6 & $1.12-0.0$ & $\ldots$ & $2443 \pm 71$ & $\ldots$ & \\
\hline $80: 77$................ & 33822.02 & -352231.6 & $1.42 \pm 0.08$ & $22.92 \pm 0.04$ & $1257 \pm 40$ & $1269 \pm 36$ & \\
\hline $80: 79$.............. & 33826.25 & $\begin{array}{lll}-35 & 22 & 24.7\end{array}$ & $1.87 \pm 0.11$ & $22.54 \pm 0.06$ & $1174 \pm 42$ & $\ldots$ & \\
\hline $80: 83 \ldots \ldots \ldots \ldots$ & 33823.88 & $-3522 \quad 12.4$ & $1.91 \pm 0.06$ & $21.74 \pm 0.03$ & $1614 \pm 24$ & $1581 \pm 20$ & \\
\hline $80: 86 \ldots \ldots \ldots . . .$. & 33818.50 & -352203.6 & $1.83 \pm 0.04$ & $21.51 \pm 0.02$ & $1248 \pm 21$ & $1287 \pm 23$ & \\
\hline $80: 88 \ldots \ldots \ldots \ldots$ & 33820.86 & -352156.0 & $1.03 \pm 0.06$ & $22.79 \pm 0.04$ & $653 \pm 59$ & $750 \pm 27$ & \\
\hline $80: 90 \ldots \ldots \ldots \ldots$ & 33817.53 & $\begin{array}{lll}-35 & 21 & 48.8\end{array}$ & $1.13 \pm 0.06$ & $22.08 \pm 0.03$ & $1917 \pm 26$ & $2015 \pm 37$ & \\
\hline $80: 94 \ldots \ldots \ldots \ldots$ & 33815.59 & -352136.3 & $1.35 \pm 0.08$ & $22.73 \pm 0.05$ & $1811 \pm 79$ & $2035 \pm 44$ & \\
\hline $80: 96 \ldots \ldots \ldots \ldots$ & 33819.11 & $\begin{array}{llll}-35 & 21 & 27.2\end{array}$ & $1.18 \pm 0.05$ & $21.25 \pm 0.03$ & $1760 \pm 30$ & $1855 \pm 29$ & \\
\hline 80:97 ............. & 33819.86 & $\begin{array}{llll}-35 & 21 & 24.1\end{array}$ & $1.35 \pm 0.07$ & $22.53 \pm 0.04$ & $1062 \pm 48$ & $1205 \pm 36$ & \\
\hline 80:98 …......... & 33823.41 & -352120.4 & $1.87 \pm 0.14$ & $22.78 \pm 0.05$ & $1517 \pm 35$ & $1442 \pm 32$ & \\
\hline $80: 99$............... & 33823.45 & $-3521 \quad 18.0$ & $1.68 \pm 0.10$ & $22.68 \pm 0.04$ & $1621 \pm 49$ & $1823 \pm 40$ & \\
\hline $80: 103 \ldots \ldots \ldots \ldots$ & 33822.18 & -352104.4 & $1.68 \pm 0.10$ & $22.39 \pm 0.03$ & $1894 \pm 33$ & $1877 \pm 25$ & \\
\hline $80: 112 \ldots \ldots \ldots \ldots$ & 33826.94 & -352031.8 & $1.30 \pm 0.05$ & $22.13 \pm 0.02$ & $1479 \pm 49$ & $1575 \pm 37$ & \\
\hline $80: 115 \ldots \ldots \ldots \ldots$ & 33816.64 & -352022.9 & $1.40 \pm 0.04$ & $19.78 \pm 0.03$ & $1432 \pm 20$ & & \\
\hline $80: 116 \ldots \ldots \ldots . .$. & 33820.23 & $\begin{array}{lll}-35 & 20 & 19.0\end{array}$ & $1.23 \pm 0.04$ & $21.43 \pm 0.03$ & $1353 \pm 38$ & $1364 \pm 22$ & \\
\hline 80:119............ & 33821.52 & -352008.5 & $1.63 \pm 0.05$ & $21.74 \pm 0.03$ & $1416 \pm 51$ & $\ldots$ & \\
\hline $81: 2 \ldots \ldots \ldots \ldots \ldots$ & 33805.78 & -352648.6 & $1.99 \pm 0.06$ & $21.80 \pm 0.03$ & $1372 \pm 36$ & $1387 \pm 23$ & \\
\hline
\end{tabular}


TABLE 3-Continued

\begin{tabular}{|c|c|c|c|c|c|c|c|}
\hline $\begin{array}{l}\text { Identifier } \\
\text { (1) }\end{array}$ & $\begin{array}{l}\text { R.A. (J2000.0) } \\
\text { (2) }\end{array}$ & $\begin{array}{c}\text { Decl. (J2000.0) } \\
\text { (3) }\end{array}$ & $\begin{array}{l}C-R \\
\text { (4) }\end{array}$ & $\begin{array}{c}R \\
(5)\end{array}$ & $\begin{array}{c}v_{\mathrm{c}} \\
\left(\mathrm{km} \mathrm{s}^{-1}\right) \\
(6)\end{array}$ & $\begin{array}{c}v_{l} \\
\left(\mathrm{~km} \mathrm{~s}^{-1}\right) \\
(7)\end{array}$ & $\begin{array}{c}\text { Comments } \\
\text { (8) }\end{array}$ \\
\hline $81: 3 \ldots \ldots \ldots \ldots . . .$. & 33803.23 & -352644.6 & $1.25 \pm 0.04$ & $21.66 \pm 0.03$ & $1797 \pm 82$ & $\ldots$ & \\
\hline 81:5 .................. & 33807.05 & -352639.8 & $1.41 \pm 0.08$ & $22.44 \pm 0.04$ & $858 \pm 47$ & $1285 \pm 45$ & \\
\hline 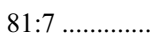 & 33810.20 & -352632.2 & $1.44 \pm 0.06$ & $21.67 \pm 0.03$ & $965 \pm 42$ & $1088 \pm 33$ & $82: 40:: 1010 \pm 37$ \\
\hline 81:8 ................ & 33803.04 & -352629.1 & $1.26 \pm 0.03$ & $21.11 \pm 0.03$ & $1570 \pm 42$ & $\ldots$ & $82: 22:: 1755 \pm 34$ \\
\hline 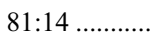 & 33803.63 & -352605.8 & $1.81 \pm 0.05$ & $21.81 \pm 0.02$ & $1403 \pm 41$ & $1399 \pm 33$ & $82: 24:: 1392 \pm 33$ \\
\hline 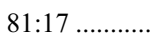 & 33802.36 & -352557.3 & $1.29 \pm 0.04$ & $21.94 \pm 0.02$ & $1109 \pm 62$ & $1055 \pm 49$ & \\
\hline 81:19 ................. & 33813.94 & -352552.2 & $\ldots$ & $\ldots$ & $1937 \pm 34$ & $1983 \pm 23$ & \\
\hline 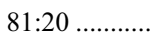 & 33807.32 & -352548.5 & $1.85 \pm 0.04$ & $21.17 \pm 0.03$ & $1624 \pm 30$ & $1671 \pm 29$ & \\
\hline 81:23 ............ & 33812.09 & -352537.3 & $1.56 \pm 0.04$ & $21.59 \pm 0.02$ & $1523 \pm 26$ & $1488 \pm 27$ & \\
\hline 81:24 ............... & 33810.05 & -352533.5 & $1.33 \pm 0.08$ & $22.67 \pm 0.04$ & $1397 \pm 53$ & $\ldots$ & \\
\hline 81:27 ...................... & 33805.19 & -352519.7 & $1.31 \pm 0.05$ & $22.01 \pm 0.02$ & $1806 \pm 41$ & $1702 \pm 34$ & \\
\hline $81: 34 \ldots \ldots \ldots . .$. & 33802.87 & -352458.0 & $1.14 \pm 0.04$ & $21.28 \pm 0.03$ & $1230 \pm 34$ & $1168 \pm 21$ & \\
\hline $81: 35 \ldots \ldots \ldots . .$. & 33811.12 & -352454.0 & $1.43 \pm 0.09$ & $22.09 \pm 0.02$ & $1874 \pm 33$ & $1920 \pm 31$ & \\
\hline $81: 36 \ldots \ldots \ldots . . .$. & 33809.46 & -352450.6 & $1.38 \pm 0.05$ & $21.96 \pm 0.03$ & $1720 \pm 46$ & $1581 \pm 24$ & \\
\hline 81:39 ................ & 33809.73 & -352437.2 & $1.38 \pm 0.03$ & $20.94 \pm 0.02$ & $1526 \pm 23$ & $1547 \pm 20$ & \\
\hline 81:40 .............. & 33812.59 & -352431.6 & $1.95 \pm 0.09$ & $22.32 \pm 0.03$ & $1649 \pm 43$ & $\ldots$ & \\
\hline $81: 41 \ldots \ldots . . . . . .$. & 33807.10 & -352429.0 & $1.91 \pm 0.04$ & $20.95 \pm 0.02$ & $1276 \pm 31$ & $1280 \pm 20$ & \\
\hline 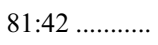 & 33810.30 & -352427.1 & $1.39 \pm 0.06$ & $22.70 \pm 0.04$ & $1771 \pm 68$ & $\ldots$ & \\
\hline 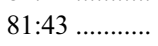 & 33807.89 & -352423.6 & $1.38 \pm 0.04$ & $21.90 \pm 0.02$ & $1656 \pm 55$ & $1653 \pm 31$ & \\
\hline 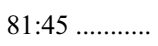 & 33806.92 & $-35 \quad 24 \quad 15.4$ & $1.34 \pm 0.03$ & $21.60 \pm 0.03$ & $1868 \pm 46$ & .. & \\
\hline $81: 46 \ldots \ldots \ldots . . . .$. & 33809.76 & $\begin{array}{lll}-35 & 24 & 09.3\end{array}$ & $1.29 \pm 0.03$ & $21.42 \pm 0.02$ & $1837 \pm 31$ & $1815 \pm 22$ & \\
\hline 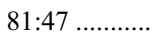 & 33810.37 & -352406.0 & $1.60 \pm 0.02$ & $19.17 \pm 0.01$ & $1582 \pm 22$ & $1638 \pm 18$ & \\
\hline 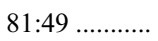 & 33808.53 & -352355.6 & $1.93 \pm 0.15$ & $21.98 \pm 0.04$ & $1363 \pm 42$ & $1334 \pm 40$ & \\
\hline 81:50 ................ & 33808.55 & -352352.5 & $\ldots$ & $\ldots$ & $1682 \pm 42$ & $1677 \pm 35$ & \\
\hline $81: 52 \ldots \ldots \ldots . .$. & 33809.14 & -352346.9 & $\ldots$ & $\ldots$ & $1446 \pm 24$ & $1520 \pm 17$ & \\
\hline 81:54 ................ & 33811.01 & -352338.4 & $\ldots$ & $\ldots$ & $1354 \pm 22$ & $1367 \pm 24$ & \\
\hline 81:55 .................. & 33813.67 & $-3523 \quad 34.9$ & $\ldots$ & $\ldots$ & $1976 \pm 61$ & $1642 \pm 43$ & \\
\hline $81: 57$.................... & 33804.69 & $-35 \quad 2325.4$ & $1.69 \pm 0.12$ & $22.42 \pm 0.05$ & $1364 \pm 57$ & $1325 \pm 36$ & \\
\hline 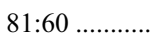 & 33809.04 & -352311.7 & $1.83 \pm 0.08$ & $22.36 \pm 0.04$ & $1452 \pm 37$ & $\ldots$ & \\
\hline 81:64 .............. & 33804.80 & -352256.6 & $1.22 \pm 0.03$ & $21.32 \pm 0.02$ & $1433 \pm 38$ & $\ldots$ & \\
\hline 81:66 ..................... & 33806.29 & -352248.7 & $1.22 \pm 0.03$ & $21.32 \pm 0.02$ & $1689 \pm 58$ & $1707 \pm 32$ & \\
\hline $81: 74 \ldots \ldots \ldots . . .$. & 33807.02 & $\begin{array}{lll}-35 & 22 & 22.3\end{array}$ & $1.26 \pm 0.07$ & $22.52 \pm 0.04$ & $1301 \pm 53$ & $1454 \pm 37$ & \\
\hline 81:75 ............... & 33814.74 & $-3522 \quad 18.7$ & $1.74 \pm 0.12$ & $22.78 \pm 0.04$ & $995 \pm 47$ & $\ldots$ & \\
\hline 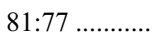 & 33812.27 & -352211.5 & $1.74 \pm 0.05$ & $21.98 \pm 0.02$ & $1347 \pm 33$ & $1296 \pm 30$ & \\
\hline 81:78 ................ & 33803.17 & $\begin{array}{lll}-35 & 22 & 08.1\end{array}$ & $1.30 \pm 0.05$ & $21.59 \pm 0.03$ & $1408 \pm 56$ & $1510 \pm 53$ & \\
\hline 81:81 ..................... & 33807.72 & -352159.5 & $1.84 \pm 0.03$ & $21.15 \pm 0.01$ & $1547 \pm 59$ & $1584 \pm 22$ & \\
\hline 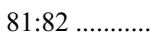 & 33813.28 & -352153.0 & $1.77 \pm 0.05$ & $21.30 \pm 0.03$ & $1086 \pm 34$ & $1168 \pm 22$ & \\
\hline 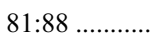 & 33812.52 & -352127.3 & $2.06 \pm 0.14$ & $22.61 \pm 0.05$ & $1496 \pm 47$ & $\ldots$ & \\
\hline 81:98 ............. & 33807.60 & -352050.0 & $1.27 \pm 0.03$ & $21.39 \pm 0.02$ & $1534 \pm 48$ & $1485 \pm 23$ & \\
\hline $81: 107$........... & 33813.35 & -352021.0 & $1.85 \pm 0.05$ & $21.73 \pm 0.03$ & $1252 \pm 26$ & $1253 \pm 23$ & \\
\hline $81: 109 \ldots \ldots . . . . . .$. & 33809.99 & -352015.1 & $1.55 \pm 0.08$ & $22.25 \pm 0.03$ & $1555 \pm 63$ & $1526 \pm 37$ & \\
\hline 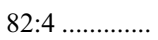 & 33757.41 & -352621.9 & $1.35 \pm 0.05$ & $21.97 \pm 0.03$ & $2149 \pm 46$ & $2155 \pm 24$ & \\
\hline 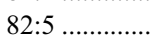 & 33757.85 & -352505.7 & $1.95 \pm 0.05$ & $21.57 \pm 0.03$ & $1063 \pm 23$ & $1003 \pm 29$ & \\
\hline 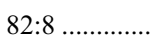 & 33758.68 & $-35 \quad 2559.2$ & $1.46 \pm 0.05$ & $22.04 \pm 0.02$ & $1186 \pm 56$ & $1258 \pm 30$ & \\
\hline 82:13 ................ & 33800.61 & -352649.8 & $1.29 \pm 0.06$ & $22.33 \pm 0.03$ & $1917 \pm 60$ & $1974 \pm 33$ & \\
\hline 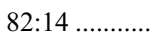 & 33801.04 & -352625.2 & $1.23 \pm 0.03$ & $21.16 \pm 0.02$ & $1134 \pm 39$ & $1111 \pm 20$ & \\
\hline 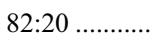 & 33802.64 & $\begin{array}{lll}-35 & 25 & 04.1\end{array}$ & $1.29 \pm 0.05$ & $22.13 \pm 0.03$ & $1328 \pm 82$ & $1424 \pm 44$ & \\
\hline 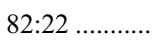 & 33803.13 & -352629.4 & $1.26 \pm 0.03$ & $21.11 \pm 0.03$ & $1939 \pm 26$ & $1944 \pm 20$ & $81: 8$ \\
\hline 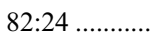 & 33803.72 & -352606.1 & $1.81 \pm 0.05$ & $21.81 \pm 0.02$ & $1380 \pm 24$ & $1424 \pm 27$ & $81: 14$ \\
\hline 82:29 ................ & 33805.73 & -352646.4 & $2.02 \pm 0.06$ & $21.77 \pm 0.03$ & $1441 \pm 32$ & $1552 \pm 29$ & \\
\hline 82:35 .............. & 33808.57 & -352436.7 & $1.80 \pm 0.10$ & $22.39 \pm 0.04$ & $1493 \pm 49$ & $1549 \pm 50$ & \\
\hline $82: 40 \ldots \ldots \ldots . . .$. & 33810.29 & -352632.5 & $1.44 \pm 0.06$ & $21.67 \pm 0.03$ & $1054 \pm 32$ & $1097 \pm 28$ & $81: 7$ \\
\hline 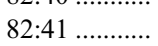 & 33810.62 & -352436.8 & $1.19 \pm 0.04$ & $22.13 \pm 0.02$ & $1166 \pm 150$ & $1309 \pm 25$ & \\
\hline 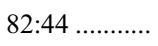 & 33811.85 & -352645.4 & $1.43 \pm 0.08$ & $22.42 \pm 0.04$ & $727 \pm 41$ & $793 \pm 41$ & \\
\hline 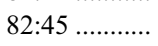 & 33812.32 & -352627.7 & $1.70 \pm 0.19$ & $21.77 \pm 0.02$ & $1513 \pm 37$ & $1568 \pm 30$ & \\
\hline $82: 50 \ldots \ldots \ldots . . .$. & 33815.02 & -352621.9 & $1.55 \pm 0.06$ & $21.76 \pm 0.03$ & $885 \pm 66$ & $827 \pm 49$ & \\
\hline 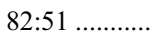 & 33815.39 & -352530.4 & $1.17 \pm 0.04$ & $21.51 \pm 0.03$ & $1933 \pm 75$ & $1735 \pm 35$ & \\
\hline $82: 52 \ldots \ldots \ldots . .$. & 33815.61 & -352644.3 & $1.53 \pm 0.04$ & $21.58 \pm 0.02$ & $1152 \pm 29$ & $1256 \pm 31$ & \\
\hline $82: 54 \ldots \ldots \ldots . . .$. & 33816.25 & -352530.6 & $1.78 \pm 0.07$ & $21.82 \pm 0.03$ & $1726 \pm 37$ & $1638 \pm 31$ & \\
\hline $82: 56 \ldots \ldots \ldots . .$. & 33817.04 & -352553.3 & $1.69 \pm 0.07$ & $22.27 \pm 0.04$ & $1998 \pm 45$ & $1862 \pm 34$ & \\
\hline $82: 57$..................... & 33817.23 & -352631.1 & $1.65 \pm 0.02$ & $20.44 \pm 0.02$ & $1232 \pm 22$ & $1248 \pm 17$ & \\
\hline $82: 59 \ldots \ldots \ldots . . .$. & 33818.36 & -352516.6 & $1.71 \pm 0.11$ & $22.61 \pm 0.07$ & $1308 \pm 40$ & $1350 \pm 34$ & \\
\hline 82:62 ............ & 33819.49 & -352611.2 & $1.73 \pm 0.06$ & $21.59 \pm 0.03$ & $1059 \pm 36$ & $1096 \pm 39$ & \\
\hline $82: 65 \ldots \ldots \ldots . . . .$. & 33820.45 & $-35 \quad 2507.6$ & $2.01 \pm 0.13$ & $22.27 \pm 0.04$ & $1731 \pm 64$ & $1708 \pm 49$ & \\
\hline
\end{tabular}


TABLE 3-Continued

\begin{tabular}{|c|c|c|c|c|c|c|c|}
\hline $\begin{array}{l}\text { Identifier } \\
\text { (1) }\end{array}$ & $\begin{array}{l}\text { R.A. (J2000.0) } \\
\text { (2) }\end{array}$ & $\begin{array}{c}\text { Decl. (J2000.0) } \\
\text { (3) }\end{array}$ & $\begin{array}{l}C-R \\
\text { (4) }\end{array}$ & $\begin{array}{c}R \\
(5)\end{array}$ & $\begin{array}{c}v_{\mathrm{c}} \\
\left(\mathrm{km} \mathrm{s}^{-1}\right) \\
(6)\end{array}$ & $\begin{array}{c}v_{l} \\
\left(\mathrm{~km} \mathrm{~s}^{-1}\right) \\
(7)\end{array}$ & $\begin{array}{c}\text { Comments } \\
\text { (8) }\end{array}$ \\
\hline 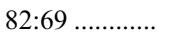 & 33821.58 & -352546.6 & $1.63 \pm 0.09$ & $22.18 \pm 0.03$ & $1347 \pm 49$ & $1241 \pm 31$ & \\
\hline $82: 70 \ldots \ldots \ldots \ldots$ & 33821.84 & $\begin{array}{lll}-35 & 25 & 15.2\end{array}$ & $1.91 \pm 0.05$ & $21.29 \pm 0.02$ & $1572 \pm 28$ & $1572 \pm 25$ & $80: 30$ \\
\hline 82:71 ............. & 33822.09 & $\begin{array}{lll}-35 & 25 & 29.3\end{array}$ & $1.63 \pm 0.10$ & $22.38 \pm 0.03$ & $1772 \pm 32$ & $1870 \pm 25$ & \\
\hline $82: 73 \ldots \ldots \ldots \ldots$ & 33822.69 & -352546.7 & $1.32 \pm 0.07$ & $22.17 \pm 0.02$ & $1563 \pm 116$ & $1702 \pm 36$ & \\
\hline $82: 74 \ldots \ldots \ldots \ldots$ & 33823.19 & -352549.8 & $1.58 \pm 0.13$ & $22.67 \pm 0.06$ & $1664 \pm 54$ & $\ldots$ & \\
\hline $82: 75 \ldots \ldots \ldots \ldots$ & 33823.49 & $\begin{array}{lll}-35 & 2522.2\end{array}$ & $1.44 \pm 0.09$ & $22.51 \pm 0.04$ & $1450 \pm 74$ & $\ldots$ & \\
\hline $82: 76 \ldots \ldots \ldots \ldots$ & 33823.72 & -352533.5 & $1.05 \pm 0.06$ & $22.52 \pm 0.04$ & $1404 \pm 98$ & $1613 \pm 29$ & \\
\hline 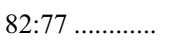 & 33824.05 & -352508.1 & $1.88 \pm 0.08$ & $22.05 \pm 0.03$ & $1623 \pm 48$ & $\ldots$ & \\
\hline $82: 78 \ldots \ldots \ldots \ldots$ & 33824.38 & -352532.5 & $1.17 \pm 0.10$ & $21.76 \pm 0.03$ & $1376 \pm 92$ & $1998 \pm 44$ & \\
\hline $82: 80 \ldots \ldots \ldots \ldots$ & 33824.86 & -352525.9 & $1.51 \pm 0.08$ & $22.48 \pm 0.04$ & $1471 \pm 62$ & $1487 \pm 25$ & \\
\hline $82: 82 \ldots \ldots \ldots \ldots$ & 33825.86 & -352456.6 & $2.02 \pm 0.09$ & $22.19 \pm 0.03$ & $1428 \pm 56$ & $1407 \pm 38$ & \\
\hline $82: 83 \ldots \ldots \ldots . . .$. & 33826.11 & -352437.2 & $1.78 \pm 0.04$ & $21.51 \pm 0.02$ & $1536 \pm 39$ & $1599 \pm 29$ & \\
\hline $82: 84 \ldots \ldots \ldots \ldots$ & 33826.52 & -352425.4 & $1.68 \pm 0.03$ & $20.73 \pm 0.01$ & $1938 \pm 41$ & $1939 \pm 22$ & \\
\hline $84: 6 \ldots \ldots \ldots \ldots . . .$. & 33849.38 & -353402.6 & $1.81 \pm 0.17$ & $22.96 \pm 0.05$ & $1894 \pm 52$ & $1488 \pm 37$ & \\
\hline $84: 8 \ldots \ldots \ldots \ldots . . .$. & 33851.27 & -353356.7 & $1.83 \pm 0.08$ & $22.80 \pm 0.04$ & $1483 \pm 66$ & $\ldots$ & \\
\hline $84: 10 \ldots \ldots \ldots \ldots$ & 33854.85 & -353348.3 & $1.38 \pm 0.04$ & $22.35 \pm 0.03$ & $1780 \pm 29$ & $\ldots$ & \\
\hline $84: 11 \ldots \ldots \ldots \ldots . .$. & 33859.34 & -353344.0 & $1.50 \pm 0.04$ & $21.56 \pm 0.03$ & $2059 \pm 28$ & $1928 \pm 26$ & \\
\hline $84: 30 \ldots \ldots \ldots \ldots$ & 33858.30 & -353229.1 & $2.03 \pm 0.06$ & $21.60 \pm 0.02$ & $1741 \pm 31$ & $1732 \pm 18$ & \\
\hline $84: 31 \ldots \ldots \ldots \ldots$ & 33852.37 & -353223.9 & $1.96 \pm 0.07$ & $21.90 \pm 0.02$ & $1407 \pm 36$ & $1353 \pm 27$ & \\
\hline $84: 33 \ldots \ldots \ldots \ldots$ & 33854.98 & $\begin{array}{lll}-35 & 32 & 16.1\end{array}$ & $1.35 \pm 0.05$ & $22.13 \pm 0.04$ & $1359 \pm 62$ & $1232 \pm 27$ & \\
\hline $84: 35$............... & 33857.03 & -353208.3 & $1.71 \pm 0.03$ & $21.15 \pm 0.02$ & $1344 \pm 27$ & $1261 \pm 33$ & \\
\hline $84: 39$............... & 33854.03 & -353158.2 & $1.74 \pm 0.02$ & $20.61 \pm 0.01$ & $1487 \pm 15$ & $1418 \pm 24$ & \\
\hline $84: 42 \ldots \ldots \ldots \ldots . .$. & 33857.43 & -353142.9 & $1.77 \pm 0.12$ & $22.72 \pm 0.04$ & $1570 \pm 46$ & $2484 \pm 33$ & \\
\hline $84: 49$............... & 33854.39 & -353114.5 & $1.76 \pm 0.05$ & $21.46 \pm 0.02$ & $891 \pm 27$ & $973 \pm 27$ & \\
\hline $84: 53 \ldots \ldots \ldots \ldots$ & 33853.13 & -353101.9 & $1.39 \pm 0.04$ & $21.92 \pm 0.02$ & $1784 \pm 49$ & $1733 \pm 25$ & \\
\hline $84: 55$............. & 33855.57 & $-35 \quad 3052.2$ & $1.20 \pm 0.04$ & $21.98 \pm 0.03$ & $2182 \pm 106$ & $2107 \pm 30$ & \\
\hline 84:60 ............. & 33900.68 & -353032.8 & $1.26 \pm 0.02$ & $21.04 \pm 0.01$ & $1311 \pm 23$ & $\ldots$ & \\
\hline $84: 63 \ldots \ldots \ldots \ldots$ & 33855.37 & $\begin{array}{lll}-35 & 30 & 22.2\end{array}$ & $1.13 \pm 0.09$ & $22.87 \pm 0.06$ & $1076 \pm 37$ & $283 \pm 23$ & \\
\hline $84: 64 \ldots \ldots \ldots \ldots . .$. & 33854.70 & -353016.6 & $1.35 \pm 0.03$ & $21.02 \pm 0.02$ & $1067 \pm 37$ & $949 \pm 30$ & \\
\hline $84: 65 \ldots \ldots \ldots \ldots .$. & 33858.25 & -353013.6 & $1.62 \pm 0.03$ & $21.43 \pm 0.02$ & $1197 \pm 34$ & $1073 \pm 35$ & \\
\hline $84: 68 \ldots \ldots \ldots \ldots . . .$. & 33859.06 & -352954.6 & $1.33 \pm 0.07$ & $22.23 \pm 0.02$ & $1948 \pm 36$ & $1269 \pm 32$ & \\
\hline $84: 72 \ldots \ldots \ldots \ldots$ & 33854.65 & -352944.8 & $1.42 \pm 0.02$ & $20.15 \pm 0.01$ & $1699 \pm 19$ & $1705 \pm 20$ & \\
\hline $84: 73 \ldots \ldots \ldots \ldots$ & 33851.70 & -352940.0 & $1.80 \pm 0.10$ & $22.54 \pm 0.04$ & $2039 \pm 72$ & $2023 \pm 28$ & \\
\hline $84: 74 \ldots \ldots \ldots \ldots . .$. & 33855.00 & -352936.0 & $1.49 \pm 0.06$ & $21.72 \pm 0.02$ & $1574 \pm 25$ & $\ldots$ & \\
\hline $84: 80 \ldots \ldots \ldots \ldots$ & 33854.75 & -352916.3 & $1.18 \pm 0.05$ & $22.31 \pm 0.03$ & $1936 \pm 54$ & $1867 \pm 50$ & \\
\hline 84:94 _.............. & 33856.50 & -352829.3 & $1.61 \pm 0.02$ & $20.65 \pm 0.01$ & $1366 \pm 23$ & $1345 \pm 25$ & \\
\hline $84: 96 \ldots \ldots \ldots \ldots$ & 33857.48 & -352819.7 & $1.23 \pm 0.06$ & $22.43 \pm 0.03$ & $1283 \pm 45$ & $896 \pm 57$ & \\
\hline $84: 110 \ldots \ldots \ldots \ldots$ & 33851.64 & -352735.3 & $1.40 \pm 0.03$ & $21.37 \pm 0.02$ & $1154 \pm 30$ & $1139 \pm 44$ & \\
\hline 84:104 .............. & 33856.18 & -352754.6 & $1.46 \pm 0.04$ & $21.39 \pm 0.02$ & $1739 \pm 50$ & 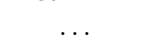 & \\
\hline $86: 4 \ldots \ldots \ldots \ldots \ldots$ & 33834.35 & -353359.3 & $1.62 \pm 0.03$ & $21.18 \pm 0.01$ & $1502 \pm 17$ & $1567 \pm 13$ & \\
\hline $86: 6 \ldots \ldots \ldots \ldots .$. & 33834.12 & -353354.6 & $1.25 \pm 0.02$ & $21.12 \pm 0.01$ & $1503 \pm 39$ & $1540 \pm 16$ & \\
\hline $86: 9$................. & 33839.14 & -353341.4 & $2.06 \pm 0.04$ & $21.33 \pm 0.02$ & $1491 \pm 25$ & $1475 \pm 17$ & \\
\hline $86: 10 \ldots \ldots \ldots \ldots$ & 33838.63 & -353335.7 & $1.89 \pm 0.04$ & $21.35 \pm 0.02$ & $1444 \pm 23$ & $1627 \pm 21$ & \\
\hline $86: 12 \ldots \ldots \ldots \ldots$ & 33838.10 & -353327.5 & $1.46 \pm 0.07$ & $21.99 \pm 0.03$ & $1861 \pm 24$ & $1887 \pm 19$ & \\
\hline $86: 13 \ldots \ldots \ldots . . .$. & 33832.09 & -353324.8 & $2.03 \pm 0.09$ & $21.96 \pm 0.03$ & $1580 \pm 23$ & $1583 \pm 26$ & \\
\hline $86: 19 \ldots \ldots \ldots \ldots$ & 33838.92 & -353258.3 & $\ldots$ & $\ldots$ & $1644 \pm 23$ & $\ldots$ & \\
\hline $86: 24 \ldots \ldots \ldots \ldots$ & 33835.96 & -353236.9 & $1.59 \pm 0.05$ & $22.04 \pm 0.03$ & $1471 \pm 17$ & $1384 \pm 19$ & \\
\hline $86: 26 \ldots \ldots \ldots . . .$. & 33833.39 & $\begin{array}{lll}-35 & 32 & 29.5\end{array}$ & $1.92 \pm 0.04$ & $21.10 \pm 0.02$ & $1862 \pm 15$ & $1898 \pm 16$ & \\
\hline $86: 27 \ldots \ldots \ldots \ldots$ & 33837.61 & -353226.0 & $1.47 \pm 0.09$ & $22.52 \pm 0.04$ & $1393 \pm 56$ & $1345 \pm 32$ & \\
\hline $86: 29 \ldots \ldots \ldots \ldots$ & 33836.09 & $\begin{array}{lll}-35 & 32 & 17.3\end{array}$ & $1.76 \pm 0.07$ & $21.99 \pm 0.03$ & $1371 \pm 16$ & $\ldots$ & \\
\hline $86: 33 \ldots \ldots \ldots \ldots$ & 33839.23 & -353201.7 & $2.01 \pm 0.06$ & $22.22 \pm 0.02$ & $1636 \pm 27$ & $1687 \pm 28$ & \\
\hline $86: 37 \ldots \ldots \ldots \ldots$ & 33833.57 & -353151.4 & $1.20 \pm 0.08$ & $22.69 \pm 0.03$ & $1805 \pm 31$ & $1710 \pm 19$ & \\
\hline $86: 39 \ldots \ldots \ldots \ldots$ & 33840.21 & -353145.4 & $1.82 \pm 0.04$ & $21.30 \pm 0.02$ & $1487 \pm 14$ & $1385 \pm 17$ & \\
\hline $86: 41 \ldots \ldots \ldots \ldots$ & 33840.87 & -353139.7 & $1.72 \pm 0.09$ & $22.42 \pm 0.03$ & $1811 \pm 37$ & $1809 \pm 25$ & \\
\hline $86: 42 \ldots \ldots \ldots \ldots$ & 33836.75 & -353137.3 & $1.28 \pm 0.08$ & $22.57 \pm 0.04$ & $1566 \pm 30$ & $1645 \pm 16$ & \\
\hline $86: 43 \ldots \ldots \ldots \ldots$ & 33838.91 & -353132.9 & $1.37 \pm 0.06$ & $21.63 \pm 0.03$ & $1833 \pm 25$ & $1816 \pm 14$ & \\
\hline $86: 46 \ldots \ldots \ldots \ldots$ & 33839.72 & -353123.6 & $1.39 \pm 0.05$ & $22.01 \pm 0.03$ & $1423 \pm 31$ & $1520 \pm 25$ & \\
\hline $86: 48 \ldots \ldots \ldots \ldots$ & 33840.28 & -353117.9 & $1.87 \pm 0.08$ & $22.23 \pm 0.03$ & $1328 \pm 12$ & $1349 \pm 20$ & \\
\hline $86: 50 \ldots \ldots \ldots \ldots . .$. & 33829.68 & -353108.9 & $1.82 \pm 0.06$ & $21.80 \pm 0.03$ & $1513 \pm 30$ & $1428 \pm 31$ & \\
\hline 86:51 ............... & 33838.43 & $\begin{array}{lll}-35 & 31 & 06.1\end{array}$ & $1.74 \pm 0.03$ & $21.08 \pm 0.01$ & $1593 \pm 23$ & $1574 \pm 14$ & \\
\hline $86: 52 \ldots \ldots \ldots \ldots$ & 33835.64 & -353102.6 & $1.5 \pm 0.02$ & $20.65 \pm 0.01$ & $1395 \pm 18$ & $1393 \pm 14$ & \\
\hline $86: 53 \ldots \ldots \ldots \ldots$ & 33831.73 & -353059.6 & $2.04 \pm 0.09$ & $22.02 \pm 0.03$ & $1627 \pm 32$ & $1566 \pm 22$ & \\
\hline $86: 56 \ldots \ldots \ldots \ldots$ & 33834.26 & -353048.9 & $1.91 \pm 0.09$ & $22.52 \pm 0.04$ & $1985 \pm 35$ & $1998 \pm 16$ & \\
\hline $86: 58 \ldots \ldots \ldots \ldots . . .$. & 33838.24 & -353039.4 & $1.52 \pm 0.03$ & $20.99 \pm 0.02$ & $1123 \pm 18$ & $1142 \pm 16$ & \\
\hline
\end{tabular}


TABLE 3-Continued

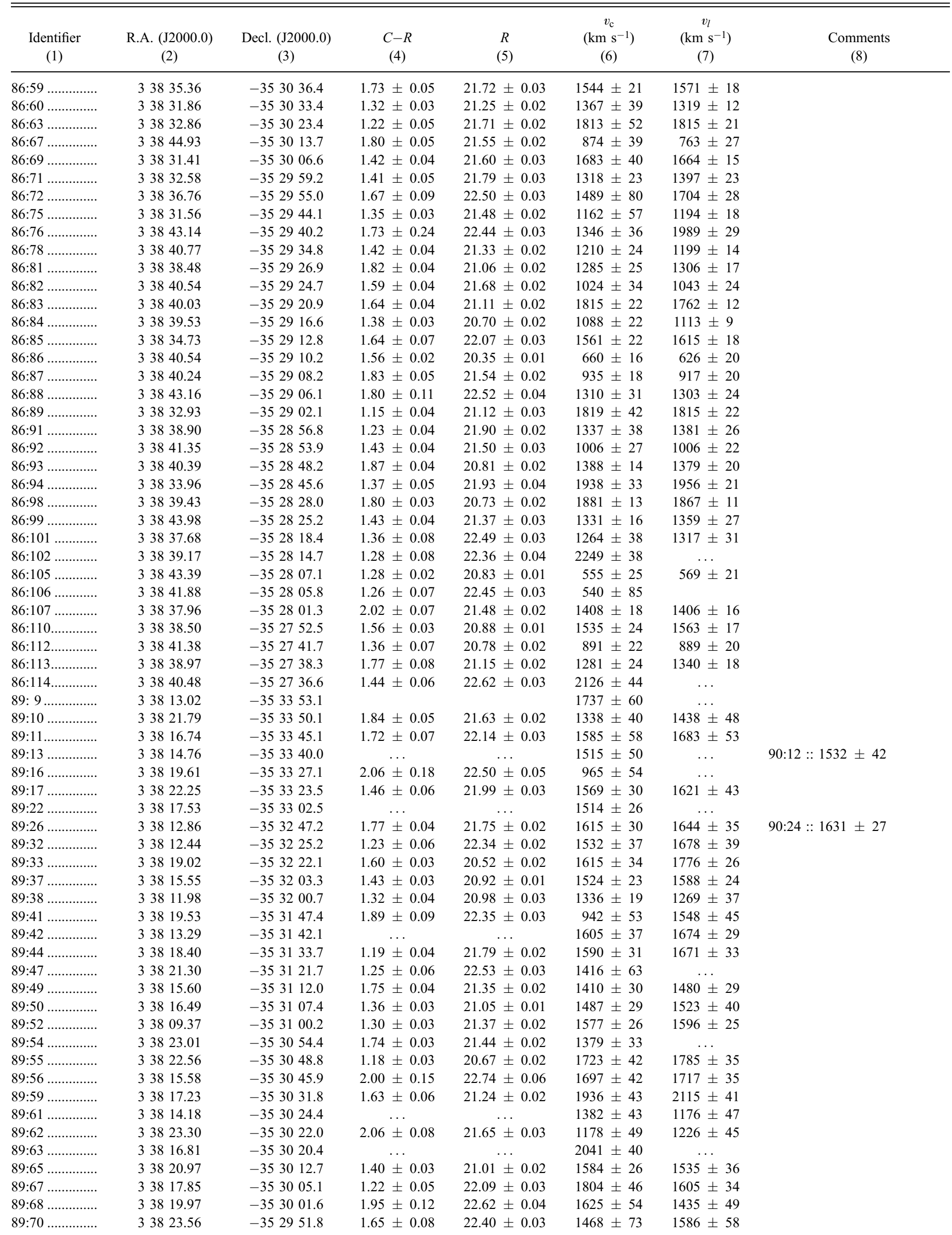


TABLE 3-Continued

\begin{tabular}{|c|c|c|c|c|c|c|c|}
\hline $\begin{array}{l}\text { Identifier } \\
\text { (1) }\end{array}$ & $\begin{array}{l}\text { R.A. (J2000.0) } \\
\text { (2) }\end{array}$ & $\begin{array}{c}\text { Decl. (J2000.0) } \\
\text { (3) }\end{array}$ & $\begin{array}{c}C-R \\
\text { (4) }\end{array}$ & $\begin{array}{c}R \\
(5)\end{array}$ & $\begin{array}{c}v_{\mathrm{c}} \\
\left(\mathrm{km} \mathrm{s}^{-1}\right) \\
(6)\end{array}$ & $\begin{array}{c}v_{l} \\
\left(\mathrm{~km} \mathrm{~s}^{-1}\right) \\
(7)\end{array}$ & $\begin{array}{c}\text { Comments } \\
\text { (8) }\end{array}$ \\
\hline $89: 73 \ldots \ldots \ldots \ldots$ & 33823.94 & -352937.3 & $1.65 \pm 0.06$ & $21.58 \pm 0.02$ & $1497 \pm 35$ & $1448 \pm 47$ & \\
\hline $89: 74 \ldots \ldots \ldots . .$. & 33816.63 & -352935.0 & $1.44 \pm 0.03$ & $21.04 \pm 0.02$ & $1664 \pm 28$ & $1544 \pm 39$ & \\
\hline $89: 76 \ldots \ldots \ldots \ldots$ & 33820.41 & -352927.7 & $1.56 \pm 0.02$ & $20.81 \pm 0.02$ & $1136 \pm 36$ & $1175 \pm 32$ & $92: 12:: 1136 \pm 36$ \\
\hline $89: 77 \ldots \ldots \ldots \ldots$ & 33821.78 & -352923.1 & $1.70 \pm 0.02$ & $20.58 \pm 0.02$ & $1648 \pm 31$ & $1630 \pm 25$ & \\
\hline $89: 78 \ldots \ldots \ldots \ldots . . .$. & 33821.19 & -352918.4 & $1.51 \pm 0.09$ & $22.42 \pm 0.05$ & $1343 \pm 47$ & $1538 \pm 55$ & $92: 10:: 1343 \pm 47$ \\
\hline $89: 84 \ldots \ldots \ldots \ldots$ & 33812.66 & -352857.1 & $1.61 \pm 0.02$ & $20.27 \pm 0.02$ & $1708 \pm 13$ & $1648 \pm 23$ & $92: 39:: 1708 \pm 13$ \\
\hline $89: 86 \ldots \ldots \ldots \ldots$ & 33821.94 & -352852.0 & $1.90 \pm 0.09$ & $22.22 \pm 0.04$ & $973 \pm 37$ & $1061 \pm 43$ & \\
\hline 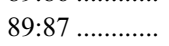 & 33822.69 & -352848.5 & $1.90 \pm 0.07$ & $21.84 \pm 0.04$ & $1435 \pm 49$ & $1561 \pm 45$ & \\
\hline $89: 90 \ldots \ldots \ldots \ldots$ & 33817.61 & -352837.6 & $1.60 \pm 0.03$ & $20.63 \pm 0.02$ & $1361 \pm 29$ & $1321 \pm 18$ & \\
\hline $89: 92 \ldots \ldots \ldots \ldots$ & 33816.62 & -352830.3 & $1.66 \pm 0.04$ & $21.54 \pm 0.02$ & $1637 \pm 57$ & $1613 \pm 35$ & \\
\hline $89: 93 \ldots \ldots \ldots \ldots$ & 33817.62 & -352826.5 & $1.52 \pm 0.03$ & $21.12 \pm 0.02$ & $1329 \pm 25$ & $1169 \pm 28$ & \\
\hline 89:94 _............ & 33815.62 & $\begin{array}{lll}-35 & 28 & 24.0\end{array}$ & $1.64 \pm 0.06$ & $21.57 \pm 0.03$ & $1573 \pm 61$ & $1761 \pm 34$ & \\
\hline 89:97 ….......... & 33816.75 & $\begin{array}{lll}-35 & 28 & 13.4\end{array}$ & $1.12 \pm 0.06$ & $22.26 \pm 0.05$ & $1571 \pm 61$ & $\ldots$ & \\
\hline $89: 99$............. & 33818.51 & -352808.5 & $1.77 \pm 0.04$ & $21.16 \pm 0.03$ & $673 \pm 51$ & $786 \pm 56$ & \\
\hline $89: 103 \ldots \ldots \ldots$ & 33815.04 & -352757.4 & $1.73 \pm 0.05$ & $21.34 \pm 0.03$ & $1711 \pm 32$ & $1759 \pm 40$ & \\
\hline $89: 105 \ldots \ldots \ldots . .$. & 33817.77 & -352750.3 & $1.56 \pm 0.08$ & $22.03 \pm 0.04$ & $859 \pm 51$ & $\ldots$ & \\
\hline 89:107 .............. & 33818.36 & -352739.6 & $1.40 \pm 0.02$ & $20.28 \pm 0.02$ & $1170 \pm 39$ & $1195 \pm 34$ & \\
\hline 89:108 ............ & 33817.69 & -352737.2 & $\ldots$ & $\ldots$ & $1484 \pm 55$ & $\ldots$ & \\
\hline $90: 2$ & 33817.14 & -353415.6 & $1.73 \pm 0.06$ & $22.04 \pm 0.02$ & $817 \pm 27$ & $1760 \pm 30$ & \\
\hline 90:4 ................ & 33813.90 & -353408.6 & $\ldots$ & $\ldots$ & $954 \pm 33$ & $1005 \pm 30$ & \\
\hline 90:9 ….............. & 33813.07 & -353352.8 & $\ldots$ & $\ldots$ & $1523 \pm 29$ & $1374 \pm 31$ & \\
\hline 90:11_............. & 33814.19 & -353345.1 & $\ldots$ & $\ldots$ & $1257 \pm 37$ & $1257 \pm 24$ & \\
\hline $90: 12 \ldots \ldots \ldots \ldots$ & 33814.80 & -353339.5 & $\ldots$ & $\ldots$ & $1549 \pm 34$ & $1510 \pm 30$ & $89: 13$ \\
\hline $90: 15 \ldots \ldots \ldots \ldots$ & 33814.77 & -353324.5 & $1.81 \pm 0.03$ & $20.67 \pm 0.02$ & $1298 \pm 31$ & $1297 \pm 20$ & \\
\hline 90:20 ….......... & 33817.53 & -353303.0 & $\ldots$ & $\ldots$ & $1451 \pm 10$ & $\ldots$ & \\
\hline 90:21 ............. & 33814.70 & -353259.6 & $\ldots$ & $\ldots$ & $1464 \pm 59$ & $\ldots$ & \\
\hline 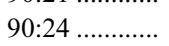 & $\begin{array}{lll}3 & 38 & 12.86\end{array}$ & -353247.2 & $1.77 \pm 0.04$ & $21.75 \pm 0.02$ & $1647 \pm 24$ & $1623 \pm 27$ & $89: 26$ \\
\hline $90: 28 \ldots \ldots \ldots \ldots$ & 33818.96 & $-3532 \quad 27.5$ & $1.42 \pm 0.11$ & $22.70 \pm 0.03$ & $1108 \pm 46$ & $1110 \pm 30$ & \\
\hline $90: 29 \ldots \ldots \ldots \ldots$ & 33812.44 & $-3532 \quad 25.2$ & $1.23 \pm 0.06$ & $22.34 \pm 0.02$ & $672 \pm 47$ & $1514 \pm 27$ & \\
\hline $90: 38 \ldots \ldots \ldots \ldots$ & $\begin{array}{lll}3 & 38 & 10.21\end{array}$ & -353158.3 & $1.99 \pm 0.05$ & $21.33 \pm 0.02$ & $1718 \pm 26$ & $1701 \pm 20$ & \\
\hline 90:41 ...................... & 33819.53 & -353147.4 & $1.89 \pm 0.09$ & $22.35 \pm 0.03$ & $1212 \pm 31$ & $\ldots$ & \\
\hline $90: 42 \ldots \ldots \ldots \ldots$ & 33809.57 & -353142.1 & $1.21 \pm 0.05$ & $22.39 \pm 0.02$ & $975 \pm 67$ & $1048 \pm 27$ & \\
\hline 90:44 ............... & 33818.34 & -353134.1 & $1.19 \pm 0.03$ & $21.79 \pm 0.03$ & $1706 \pm 32$ & & \\
\hline $90: 52 \ldots \ldots \ldots \ldots$ & 33806.48 & -353101.6 & $1.44 \pm 0.05$ & $21.50 \pm 0.03$ & $1098 \pm 23$ & $1078 \pm 28$ & \\
\hline 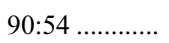 & 33818.07 & -353053.3 & $1.15 \pm 0.04$ & $22.01 \pm 0.02$ & $1593 \pm 31$ & $1643 \pm 24$ & \\
\hline 90:59 ............... & $\begin{array}{lll}3 & 38 & 17.17\end{array}$ & -353032.3 & & & $1970 \pm 20$ & & \\
\hline $90: 62 \ldots \ldots \ldots \ldots$ & 33816.44 & -353024.9 & $2.02 \pm 0.10$ & $22.11 \pm 0.03$ & $2100 \pm 30$ & $1990 \pm 20$ & \\
\hline 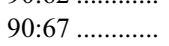 & 33817.85 & -353005.1 & $1.22 \pm 0.05$ & $22.09 \pm 0.03$ & $1556 \pm 45$ & $1559 \pm 28$ & \\
\hline 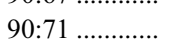 & 33818.98 & -352950.9 & $\ldots$ & $26.03-0.08$ & $2824 \pm 29$ & $2759 \pm 50$ & \\
\hline $90: 72 \ldots \ldots \ldots \ldots$ & 33815.36 & -352946.4 & $1.63 \pm 0.06$ & $22.22 \pm 0.02$ & $1311 \pm 35$ & $1095 \pm 33$ & \\
\hline $90: 74 \ldots \ldots \ldots \ldots$ & 33808.77 & -352939.4 & $1.26 \pm 0.03$ & $20.71 \pm 0.02$ & $946 \pm 20$ & $978 \pm 15$ & \\
\hline $90: 75 \ldots \ldots \ldots \ldots$ & 33817.59 & -352935.3 & $1.81 \pm 0.05$ & $21.59 \pm 0.03$ & $1349 \pm 21$ & $1320 \pm 31$ & \\
\hline $90: 76 \ldots \ldots \ldots \ldots$ & 33811.18 & -352932.0 & $1.52 \pm 0.04$ & $20.88 \pm 0.02$ & $1051 \pm 15$ & $1022 \pm 19$ & \\
\hline 90:77 ......................... & 33811.41 & -352930.0 & $1.75 \pm 0.04$ & $21.18 \pm 0.02$ & $1358 \pm 19$ & $1377 \pm 20$ & \\
\hline 90:79 ............... & 33810.47 & -352923.0 & $2.09 \pm 0.08$ & $21.86 \pm 0.02$ & $1359 \pm 28$ & $1420 \pm 34$ & \\
\hline 90:81 ............... & 33819.62 & -352918.3 & $1.69 \pm 0.13$ & $22.68 \pm 0.04$ & $1409 \pm 49$ & $\ldots$ & \\
\hline $90: 82 \ldots \ldots \ldots \ldots$ & 33808.73 & -352913.6 & $1.56 \pm 0.04$ & $21.69 \pm 0.03$ & $1152 \pm 21$ & $1102 \pm 33$ & $92: 49:: 1153 \pm 30$ \\
\hline 90:84 ............... & 33809.12 & -352905.9 & $1.91 \pm 0.05$ & $21.10 \pm 0.03$ & $1292 \pm 20$ & $1364 \pm 28$ & \\
\hline 90:86 ....................... & 33806.29 & -352857.7 & $1.62 \pm 0.05$ & $18.80 \pm 0.04$ & $1218 \pm 9$ & $1201 \pm 12$ & $\begin{array}{l}91: 93,2 \mathrm{dF}: 1254:: \\
1233 \pm 15\end{array}$ \\
\hline 90:87 ............... & 33810.43 & -352854.5 & $1.92 \pm 0.11$ & $22.26 \pm 0.04$ & $1660 \pm 47$ & & $92: 45:: 1670 \pm 48$ \\
\hline $90: 89 \ldots \ldots \ldots . .$. & 33810.95 & -352847.1 & $1.79 \pm 0.23$ & $22.75 \pm 0.05$ & $1234 \pm 71$ & $1105 \pm 54$ & \\
\hline 90:92 ................... & 33817.61 & -352837.5 & $1.60 \pm 0.03$ & $20.63 \pm 0.02$ & $1257 \pm 17$ & $1229 \pm 19$ & \\
\hline 90:93 ...................... & 33818.15 & -352834.6 & $1.77 \pm 0.15$ & $22.69 \pm 0.05$ & $719 \pm 43$ & $1489 \pm 28$ & \\
\hline 90:94 ...................... & 33816.63 & -352830.3 & $1.66 \pm 0.04$ & $21.54 \pm 0.02$ & $1484 \pm 25$ & $1507 \pm 17$ & \\
\hline $90: 95 \ldots \ldots \ldots \ldots$ & 33817.63 & -352826.7 & $1.52 \pm 0.03$ & $21.12 \pm 0.02$ & $1260 \pm 38$ & $1248 \pm 30$ & \\
\hline $90: 100 \ldots \ldots \ldots$. & 33818.45 & $-35 \quad 2808.9$ & $1.76 \pm 0.03$ & $21.16 \pm 0.03$ & $498 \pm 15$ & 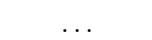 & \\
\hline 90:101 ................. & 33812.73 & -352804.8 & $1.49 \pm 0.04$ & $21.16 \pm 0.03$ & $1559 \pm 18$ & $1570 \pm 20$ & \\
\hline $90: 104 \ldots \ldots \ldots$. & 33816.70 & -352756.4 & $1.23 \pm 0.03$ & $21.44 \pm 0.02$ & $1784 \pm 30$ & $1846 \pm 27$ & \\
\hline $90: 105 \ldots \ldots \ldots$. & 33808.86 & -352753.7 & $1.87 \pm 0.05$ & $21.82 \pm 0.03$ & $1759 \pm 39$ & $1664 \pm 27$ & \\
\hline $90: 107 \ldots \ldots \ldots$. & 33816.19 & -352748.7 & $1.82 \pm 0.08$ & $22.20 \pm 0.03$ & $1170 \pm 39$ & $1185 \pm 36$ & \\
\hline 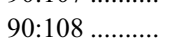 & 33818.73 & -352744.2 & $1.96 \pm 0.13$ & $22.36 \pm 0.06$ & $1484 \pm 55$ & $1598 \pm 69$ & \\
\hline 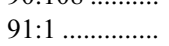 & 33802.25 & -353423.5 & $1.17 \pm 0.07$ & $22.84 \pm 0.04$ & $1008 \pm 74$ & $1049 \pm 30$ & \\
\hline 91:8 …............... & 33759.37 & -353353.3 & $1.27 \pm 0.08$ & $22.57 \pm 0.05$ & $1977 \pm 40$ & & \\
\hline
\end{tabular}


TABLE 3-Continued

\begin{tabular}{|c|c|c|c|c|c|c|c|}
\hline $\begin{array}{l}\text { Identifier } \\
\text { (1) }\end{array}$ & $\begin{array}{l}\text { R.A. (J2000.0) } \\
\text { (2) }\end{array}$ & $\begin{array}{c}\text { Decl. (J2000.0) } \\
\text { (3) }\end{array}$ & $\begin{array}{l}C-R \\
\text { (4) }\end{array}$ & $\begin{array}{c}R \\
(5)\end{array}$ & $\begin{array}{c}v_{\mathrm{c}} \\
\left(\mathrm{km} \mathrm{s}^{-1}\right) \\
(6)\end{array}$ & $\begin{array}{c}v_{l} \\
\left(\mathrm{~km} \mathrm{~s}^{-1}\right) \\
(7)\end{array}$ & $\begin{array}{c}\text { Comments } \\
\text { (8) }\end{array}$ \\
\hline 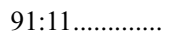 & 33807.48 & -353338.8 & $1.42 \pm 0.06$ & $22.32 \pm 0.03$ & $1106 \pm 43$ & $1053 \pm 30$ & \\
\hline 91:23 ...................... & 33759.02 & -353256.3 & $1.33 \pm 0.04$ & $21.77 \pm 0.02$ & $1917 \pm 61$ & $2073 \pm 30$ & \\
\hline 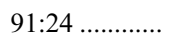 & 33755.98 & -353254.2 & $2.07 \pm 0.04$ & $21.33 \pm 0.03$ & $983 \pm 24$ & $961 \pm 29$ & \\
\hline 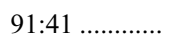 & 33756.84 & -353151.0 & $1.20 \pm 0.03$ & $20.84 \pm 0.03$ & $1472 \pm 29$ & $1545 \pm 27$ & \\
\hline 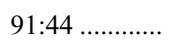 & 33804.24 & -353139.5 & $1.17 \pm 0.07$ & $22.54 \pm 0.05$ & $1853 \pm 65$ & $1942 \pm 37$ & \\
\hline 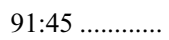 & 33751.78 & -353136.2 & $1.31 \pm 0.03$ & $21.22 \pm 0.02$ & $1410 \pm 55$ & $1465 \pm 19$ & \\
\hline 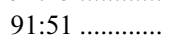 & 33757.78 & -353114.0 & $1.93 \pm 0.09$ & $22.19 \pm 0.03$ & $1344 \pm 27$ & $1395 \pm 25$ & \\
\hline 91:52 ...................... & 33804.89 & -353110.0 & $1.79 \pm 0.09$ & $22.13 \pm 0.04$ & $1630 \pm 33$ & $\ldots$ & \\
\hline 91:55 ........................ & 33804.52 & -353059.9 & $1.34 \pm 0.04$ & $21.88 \pm 0.02$ & $1327 \pm 57$ & $\ldots$ & \\
\hline 91:57 _................ & 33752.64 & -353052.4 & $1.92 \pm 0.03$ & $20.87 \pm 0.02$ & $1033 \pm 34$ & $1053 \pm 16$ & \\
\hline 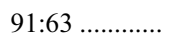 & 33802.39 & -353036.3 & $1.76 \pm 0.08$ & $22.55 \pm 0.04$ & $789 \pm 37$ & $612 \pm 28$ & \\
\hline 91:66 ................. & 33801.03 & -353025.2 & $1.35 \pm 0.05$ & $21.43 \pm 0.03$ & $1889 \pm 36$ & $1894 \pm 28$ & \\
\hline 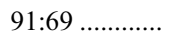 & 33755.18 & -353014.5 & $1.81 \pm 0.05$ & $21.57 \pm 0.02$ & $1287 \pm 32$ & $1282 \pm 37$ & \\
\hline 91:71 ...................... & 33804.64 & $-35: 3008.3$ & $1.63 \pm 0.03$ & $20.63 \pm 0.02$ & $1565 \pm 20$ & $1526 \pm 23$ & $9260:: 1555 \pm 24$ \\
\hline $9175 \ldots \ldots \ldots \ldots$ & 3 37:54.88 & -352953.8 & $1.98 \pm 0.06$ & $21.84 \pm 0.03$ & $1306 \pm 60$ & & $92: 90:: 1292 \pm 55$ \\
\hline 91:81 ............... & 33752.96 & -352937.6 & $1.22 \pm 0.04$ & $22.12 \pm 0.02$ & $1091 \pm 33$ & $1148 \pm 35$ & \\
\hline 91:83 ..................... & 33758.71 & -352932.4 & $1.43 \pm 0.03$ & $20.87 \pm 0.03$ & $1627 \pm 29$ & $1657 \pm 14$ & \\
\hline 91:85 ...................... & 33802.79 & -352926.1 & $1.67 \pm 0.06$ & $22.10 \pm 0.03$ & $1517 \pm 38$ & $\ldots$ & \\
\hline 91:87 ................... & 33803.89 & -352919.2 & $1.73 \pm 0.06$ & $22.26 \pm 0.02$ & $1466 \pm 54$ & $1397 \pm 40$ & \\
\hline 91:92 ..................... & 33804.27 & -352901.2 & $1.59 \pm 0.04$ & $21.51 \pm 0.03$ & $1409 \pm 37$ & $1450 \pm 22$ & $92: 62:: 1364 \pm 57$ \\
\hline 91:93 .................... & 33806.27 & -352858.7 & $1.62 \pm 0.05$ & $18.80 \pm 0.04$ & $1247 \pm 16$ & $1249 \pm 10$ & $90: 86,2 \mathrm{dF}: 1254$ \\
\hline $91: 102 \ldots \ldots . . . . . .$. & 33803.10 & -352826.1 & $1.95 \pm 0.05$ & $21.11 \pm 0.04$ & $1347 \pm 19$ & $1365 \pm 22$ & \\
\hline 91:106 _.............. & 33806.43 & $\begin{array}{lll}-35 & 28 & 16.7\end{array}$ & $1.43 \pm 0.05$ & $22.66 \pm 0.04$ & $2626 \pm 42$ & $\ldots$ & \\
\hline 91:109 _............. & 33804.39 & -352811.6 & $1.81 \pm 0.03$ & $20.82 \pm 0.02$ & $1639 \pm 19$ & $\ldots$ & \\
\hline $91: 111 \ldots \ldots \ldots . . .$. & 33801.17 & -352801.7 & $1.44 \pm 0.07$ & $22.23 \pm 0.03$ & $2395 \pm 34$ & $2489 \pm 35$ & \\
\hline 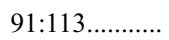 & 33808.14 & -352752.1 & $1.36 \pm 0.02$ & $20.56 \pm 0.02$ & $1805 \pm 21$ & $1833 \pm 26$ & $92: 51:: 1785 \pm 23$ \\
\hline 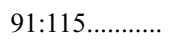 & 33804.27 & -352747.0 & $1.30 \pm 0.04$ & $21.30 \pm 0.03$ & $1571 \pm 33$ & $1559 \pm 22$ & \\
\hline 92:2 …............. & 33824.03 & -352856.4 & $1.21 \pm 0.03$ & $21.55 \pm 0.02$ & $1415 \pm 36$ & $1654 \pm 40$ & \\
\hline 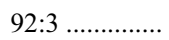 & 33823.80 & -352848.4 & $2.00 \pm 0.07$ & $21.60 \pm 0.04$ & $1735 \pm 27$ & $1428 \pm 37$ & \\
\hline $92: 4 \ldots \ldots \ldots \ldots . .$. & 33823.42 & -353048.1 & $1.25 \pm 0.04$ & $21.35 \pm 0.03$ & $814 \pm 41$ & $896 \pm 37$ & \\
\hline 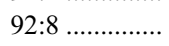 & 33822.00 & -352936.5 & $2.01 \pm 0.07$ & $21.88 \pm 0.03$ & $1256 \pm 28$ & $1197 \pm 28$ & \\
\hline 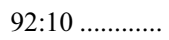 & 33821.19 & -35 2923.4 & $2.12 \pm 0.08$ & $21.81 \pm 0.03$ & $1325 \pm 46$ & $1336 \pm 16$ & $89: 78$ \\
\hline $92: 11 \ldots \ldots \ldots \ldots$ & 33820.80 & -352828.4 & $1.33 \pm 0.06$ & $22.20 \pm 0.05$ & $1581 \pm 45$ & $1580 \pm 28$ & \\
\hline 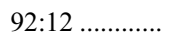 & 33820.41 & -352927.9 & $1.56 \pm 0.02$ & $20.81 \pm 0.02$ & $1256 \pm 19$ & $1252 \pm 28$ & $89: 76$ \\
\hline 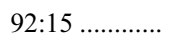 & 33819.76 & -352744.5 & $1.28 \pm 0.04$ & $21.68 \pm 0.03$ & $1652 \pm 60$ & $1619 \pm 26$ & \\
\hline 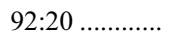 & 33818.52 & $\begin{array}{lll}-35 & 28 & 07.4\end{array}$ & $1.77 \pm 0.02$ & $21.16 \pm 0.02$ & $470 \pm 36$ & $\ldots$ & \\
\hline 92:23 ...................... & 33817.73 & -35 2914.4 & $1.91 \pm 0.13$ & $22.89 \pm 0.04$ & $1555 \pm 76$ & $\ldots$ & \\
\hline 92:28 .................. & 33815.99 & -352936.1 & $1.58 \pm 0.08$ & $22.21 \pm 0.03$ & $1579 \pm 45$ & $1290 \pm 34$ & \\
\hline 92:29 ............ & 33815.51 & -352806.3 & $1.99 \pm 0.09$ & $22.75 \pm 0.05$ & $1103 \pm 38$ & $\ldots$ & \\
\hline 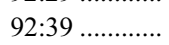 & 33812.66 & -352857.1 & $1.61 \pm 0.02$ & $20.27 \pm 0.02$ & $1605 \pm 15$ & $1626 \pm 19$ & $89: 84$ \\
\hline 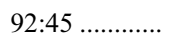 & 33810.42 & -352854.3 & $1.92 \pm 0.11$ & $22.26 \pm 0.04$ & $1679 \pm 48$ & $1553 \pm 29$ & $90: 87$ \\
\hline 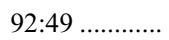 & 33808.75 & -35 2913.7 & $1.56 \pm 0.04$ & $21.69 \pm 0.03$ & $1154 \pm 38$ & $1228 \pm 31$ & $90: 82$ \\
\hline 92:51 ................ & 33808.12 & -352752.2 & $1.36 \pm 0.02$ & $20.56 \pm 0.02$ & $1764 \pm 25$ & $1777 \pm 15$ & $91: 113$ \\
\hline 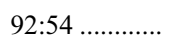 & 33807.03 & -352931.3 & $1.93 \pm 0.07$ & $22.06 \pm 0.02$ & $1907 \pm 37$ & $1861 \pm 39$ & \\
\hline 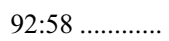 & 33805.29 & -352932.4 & $1.59 \pm 0.12$ & $22.71 \pm 0.05$ & $1033 \pm 37$ & $1955 \pm 30$ & \\
\hline 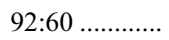 & 33804.64 & -353008.3 & $1.63 \pm 0.03$ & $20.63 \pm 0.02$ & $1544 \pm 28$ & $1468 \pm 37$ & $91: 71$ \\
\hline 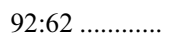 & 33804.27 & -352901.1 & $1.59 \pm 0.04$ & $21.51 \pm 0.03$ & $1319 \pm 76$ & $1571 \pm 38$ & $91: 92$ \\
\hline 92:65 ....................... & 33803.19 & -352859.7 & $1.86 \pm 0.12$ & $22.66 \pm 0.05$ & $1268 \pm 47$ & & \\
\hline $92: 74 \ldots \ldots \ldots . . .$. & 33800.16 & -353008.5 & $1.46 \pm 0.04$ & $20.57 \pm 0.04$ & $843 \pm 10$ & $849 \pm 18$ & \\
\hline 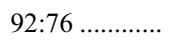 & 33759.37 & -353025.4 & $1.21 \pm 0.04$ & $21.97 \pm 0.02$ & $1827 \pm 57$ & $1746 \pm 34$ & \\
\hline 92:78 ............. & 33758.66 & -352940.9 & $1.54 \pm 0.06$ & $21.98 \pm 0.02$ & $1981 \pm 40$ & $1925 \pm 33$ & \\
\hline 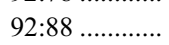 & 33755.47 & $\begin{array}{l}-352934.2 \\
-15\end{array}$ & $1.74 \pm 0.11$ & $22.65 \pm 0.05$ & $1315 \pm 49$ & $\ldots$ & \\
\hline 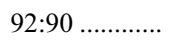 & 33754.87 & -352953.8 & $1.98 \pm 0.06$ & $21.84 \pm 0.03$ & $1281 \pm 51$ & $1502 \pm 34$ & $91: 75$ \\
\hline 92:95 ....................... & 33753.36 & -352835.7 & $1.39 \pm 0.08$ & $21.22 \pm 0.04$ & $855 \pm 36$ & $831 \pm 29$ & \\
\hline 92:97 ................. & 33752.94 & -352926.4 & $1.91 \pm 0.05$ & $21.98 \pm 0.03$ & $1756 \pm 68$ & $\ldots$ & \\
\hline 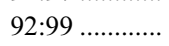 & 33752.43 & -352857.9 & $2.03 \pm 0.03$ & $20.21 \pm 0.03$ & $1431 \pm 34$ & $1468 \pm 23$ & \\
\hline
\end{tabular}

${ }^{\text {a }}$ The coordinates are tied to the USNO-A2.0 catalog. The appearance of a cluster identifier in the last column indicates that two velocities have been obtained independently on two masks. In this case the velocity, in the last column, is the mean value from the two masks. 


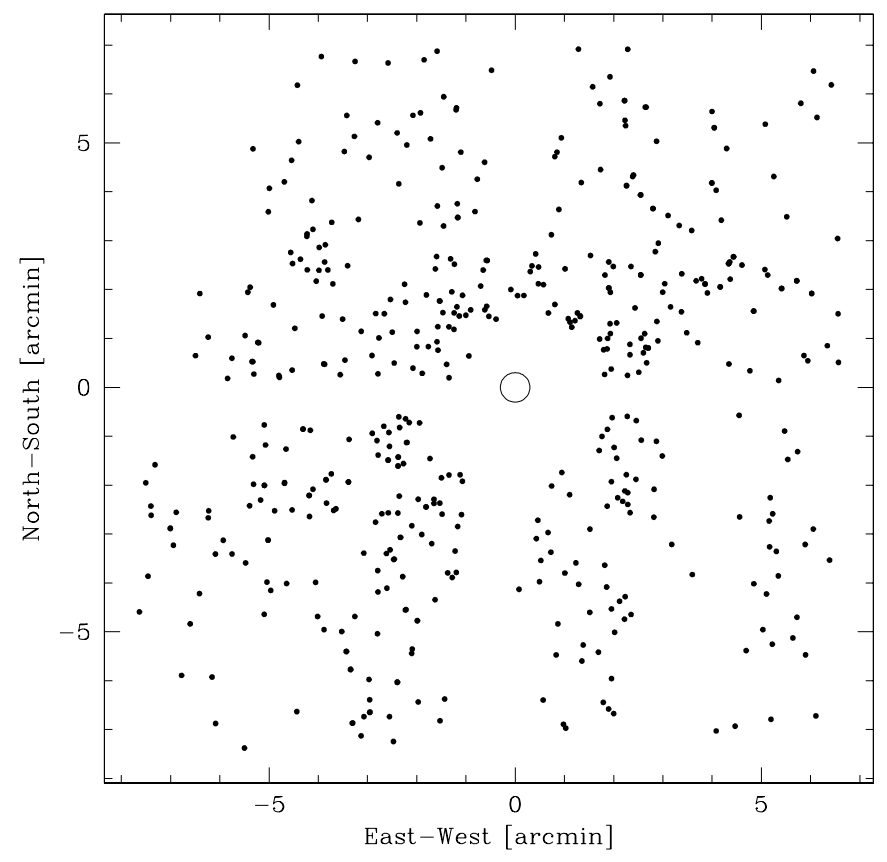

FIG. 7.-Distribution of the final cluster sample. The open circle denotes the center of NGC 1399.

do not include the photometric calibration errors that are 0.03 in $C-T 1$ and 0.02 in $T 1$. The sixth column gives the velocity determined with the correlation measurement, and the seventh column the velocity determined using direct line measurements. The uncertainties given are those returned by the respective packages used. The last column is reserved for comments. An identifier for a different cluster indicates that this cluster has been observed independently on two masks and can be identified via its number on the other mask. The spatial distribution of the whole cluster sample tabulated in Table 3 is shown in Figure 7.

Color and magnitude information are missing for some clusters. The reason is either the incomplete coverage of the field, due to the undithered gaps in the MOSAIC image, or the location of the clusters within saturated regions caused by bright nearby objects. These candidates had been selected "by eye" after all object slits on the mask had been set as described above. The velocity given is the mean value of the two correlation measurements and the error a simple mean error. We quote the mean velocity only for the first appearance of the cluster in the list.

The color and magnitude dependence of the correlation velocities are shown in Figure 8. The dynamical interpretation is given in Paper II.

Stars in our sample for which velocities have been determined are tabulated in Table 4. In some cases no correlation velocities are given, only line velocities. The reason is that our template is not particularly suited to be correlated with latetype stars, which also explains the systematically larger uncertainties in the correlation velocities.

\section{COMPARISON WITH THE LITERATURE}

Globular clusters around NGC 1399 have been spectroscopically observed earlier. The largest sample, containing 47 globular cluster velocities, has been obtained by Grillmair et al. (1994) using the Anglo-Australian Telescope with the LowDispersion Survey Spectrograph with $\approx 13 \AA$ resolution, which resulted in a velocity uncertainty of approximately $150 \mathrm{~km} \mathrm{~s}^{-1}$. Better velocities with uncertainties of approximately $100 \mathrm{~km}$ $\mathrm{s}^{-1}$ have been measured for 18 clusters by Minniti et al. (1998) with the ESO Multi-Mode Instrument at the ESO New Technology Telescope with a resolution of $7.5 \AA$. The observations done by Kissler-Patig et al. (1998) with the Low Resolution Imaging Spectrograph at Keck (resolution of 5.6 $\AA$ ) yielded the best velocities so far for 18 globular clusters with uncertainties around $35 \mathrm{~km} \mathrm{~s}^{-1}$. Some of these globular clusters were observed in our run as well, and in Table 5 our velocities are compared with the velocities determined in the earlier studies. In addition, we compiled stars that were observed in our run and are also present in the Two Degree Field (2dF) Survey (Drinkwater et al. 2001a).

The mean difference and standard deviation between our velocities and those of Kissler-Patig et al. (1998) (we always subtract the reference velocity from ours) is +101 and $58 \mathrm{~km}$ $\mathrm{s}^{-1}$, respectively. From the published uncertainties we would expect a standard deviation of approximately $60 \mathrm{~km} \mathrm{~s}^{-1}$, which agrees well. From the Grillmair et al. (1994) data we find for the mean difference and standard deviation -293 and $153 \mathrm{~km} \mathrm{~s}^{-1}$, respectively. This large difference is driven mainly by a few extremely deviating objects, probably caused by the low $\mathrm{S} / \mathrm{N}$ of Grillmair et al.'s spectra. However, in this case as well the observed and expected standard deviations are in good agreement. Regarding the stars in common with the $2 \mathrm{dF}$ survey, we find a difference of $-56 \mathrm{~km} \mathrm{~s}^{-1}$ with a standard deviation of $83 \mathrm{~km} \mathrm{~s}^{-1}$, while we would expect a standard deviation of $61 \mathrm{~km} \mathrm{~s}^{-1}$. The reason for the slight
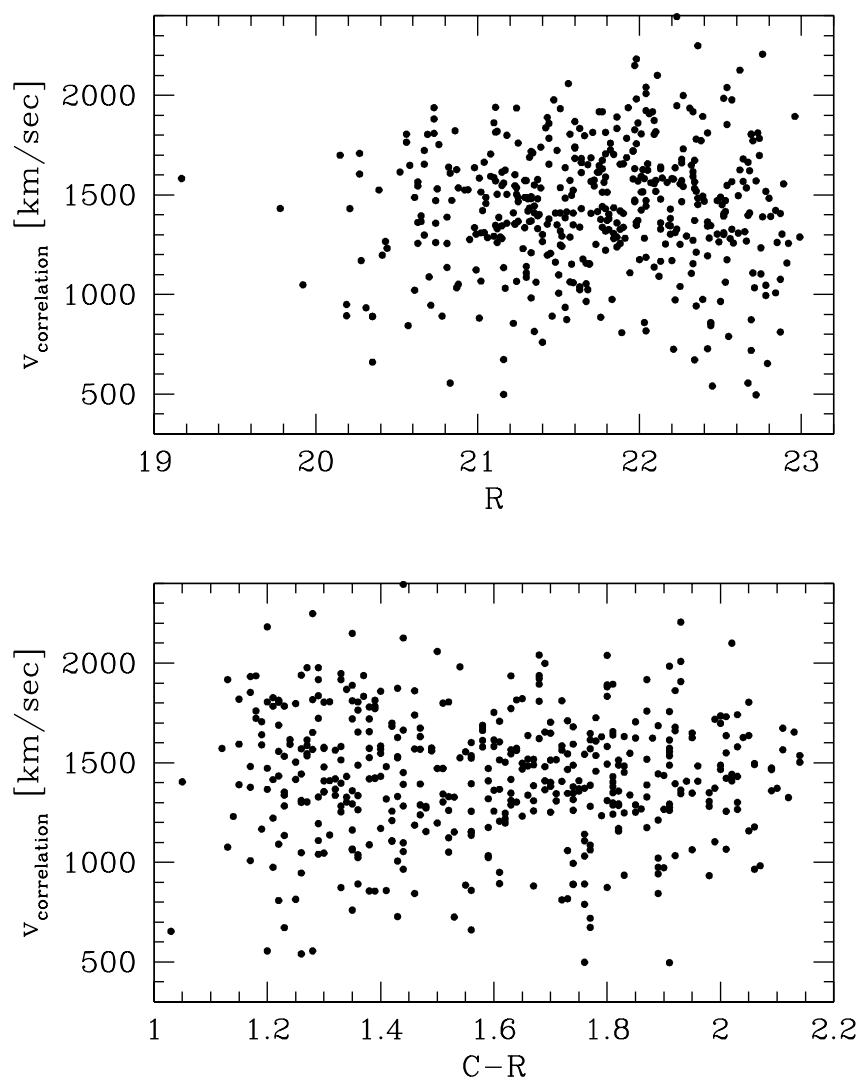

FIG. 8.-Luminosity (top) and color (bottom) dependence of the correlation velocities. No correlation is seen with luminosity; however, the scatter of blue objects is larger than that of red objects, which is due to their different dynamics explained in Paper II. 
TABLE 4

Stars in our Sample for Which Velocities Have Been Determined with Lines or Cross Correlation 3

\begin{tabular}{|c|c|c|c|c|c|c|c|}
\hline $\begin{array}{l}\text { Identifier } \\
\text { (1) }\end{array}$ & $\begin{array}{l}\text { R.A. (J2000.0) } \\
\text { (2) }\end{array}$ & $\begin{array}{l}\text { Decl. (J2000.0) } \\
\text { (3) }\end{array}$ & $\begin{array}{l}C-R \\
(4)\end{array}$ & $\begin{array}{c}R \\
(5)\end{array}$ & $\begin{array}{c}v_{c} \\
\left(\mathrm{~km} \mathrm{~s}^{-1}\right) \\
(6)\end{array}$ & $\begin{array}{c}v_{l} \\
\left(\mathrm{~km} \mathrm{~s}^{-1}\right) \\
(7)\end{array}$ & $\begin{array}{c}\text { Comments } \\
\text { (8) }\end{array}$ \\
\hline 75:15 ............... & 33859.48 & -352605.9 & $\ldots$ & $\ldots$ & $120 \pm 140$ & $\ldots$ & $78: 9$ \\
\hline 75:53 ................ & 33855.31 & -352347.9 & $\ldots$ & $\ldots$ & $114 \pm 67$ & $42 \pm 18$ & \\
\hline 75:55 ................. & 33858.17 & -352339.8 & $\ldots$ & $\ldots$ & $71 \pm 61$ & $-4 \pm 23$ & \\
\hline 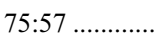 & 33857.87 & $\begin{array}{lll}-35 & 23 & 32.5\end{array}$ & $\ldots$ & $\ldots$ & $-77 \pm 90$ & $-51 \pm 31$ & \\
\hline 75:83 ................... & 33850.48 & -352213.4 & $1.78 \pm 0.03$ & $20.83 \pm 0.02$ & $145 \pm 95$ & $\ldots$ & \\
\hline 75:87 ................ & 33855.78 & -352159.4 & $0.88 \pm 0.01$ & $17.93 \pm 0.01$ & $168 \pm 26$ & $-31 \pm 22$ & \\
\hline 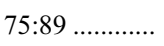 & 33851.42 & -352151.9 & $2.41 \pm 0.02$ & $17.86 \pm 0.01$ & $32 \pm 39$ & $153 \pm 15$ & \\
\hline 75:90 ................ & 33857.66 & -352147.1 & $3.12 \pm 0.04$ & $19.81 \pm 0.02$ & $39 \pm 79$ & $-77 \pm 33$ & \\
\hline 75:96 …............ & 33858.71 & -352123.4 & $2.96 \pm 0.01$ & $17.41 \pm 0.01$ & $-30 \pm 71$ & $-123 \pm 32$ & \\
\hline $76: 33 \ldots \ldots \ldots . .$. & 33846.57 & -352513.1 & $2.68 \pm 0.01$ & $16.23 \pm 0.01$ & $\ldots$ & $28 \pm 11$ & \\
\hline $76: 49 \ldots \ldots \ldots . .$. & 33841.08 & -352421.6 & $\ldots$ & $\ldots$ & $33 \pm 68$ & $-4 \pm 14$ & \\
\hline $76: 56 \ldots \ldots \ldots \ldots$ & 33837.82 & -352355.8 & $\ldots$ & $\ldots$ & $\ldots$ & $-60 \pm 23$ & \\
\hline 76:85 ................. & 33842.52 & -352221.0 & $0.73 \pm 0.01$ & $17.59 \pm 0.01$ & $\ldots$ & $120 \pm 21$ & \\
\hline $76: 87 \ldots \ldots \ldots . .$. & 33850.49 & -352213.4 & $1.78 \pm 0.03$ & $20.83 \pm 0.02$ & $83 \pm 10$ & $74 \pm 38$ & $75: 83$ \\
\hline $76: 88 \ldots \ldots \ldots \ldots$ & 33845.69 & -352210.4 & $\ldots$ & $\ldots$ & $-60 \pm 51$ & $-96 \pm 32$ & \\
\hline 76:94 ............... & 33851.44 & -352151.9 & $2.46 \pm 0.01$ & $17.77 \pm 0.01$ & $\ldots$ & $7 \pm 14$ & \\
\hline $76: 102 \ldots \ldots \ldots . .$. & 33847.33 & -352126.8 & $\ldots$ & $\ldots$ & $\ldots$ & $-7 \pm 18$ & \\
\hline 77:6 ................... & 33839.55 & -352632.1 & $\ldots$ & $\ldots$ & $4 \pm 34$ & $\ldots$ & \\
\hline 77:58 ...................... & 33842.73 & $-35 \quad 2340.2$ & $\ldots$ & $\ldots$ & $-21 \pm 68$ & $\ldots$ & \\
\hline 77:81 ............... & 33835.61 & -352228.9 & $\ldots$ & $\ldots$ & $-8 \pm 59$ & $\ldots$ & \\
\hline 77:83 ................ & 33842.52 & -352221.0 & $0.77 \pm 0.01$ & $17.71 \pm 0.01$ & $124 \pm 29$ & $131 \pm 14$ & \\
\hline 77:114.............. & 33838.09 & -352041.9 & $0.62 \pm 0.01$ & $18.24 \pm 0.01$ & $319 \pm 39$ & $318 \pm 16$ & \\
\hline 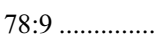 & 33859.49 & -352605.9 & $1.38 \pm 0.01$ & $21.31 \pm 0.01$ & $105 \pm 54$ & & \\
\hline $78: 24 \ldots \ldots \ldots \ldots . .$. & 33855.30 & -352348.0 & $1.51 \pm 0.05$ & $22.48 \pm 0.03$ & $109 \pm 72$ & $74 \pm 24$ & \\
\hline 78:33 ....................... & 33852.80 & $-35 \quad 2512.6$ & $1.64 \pm 0.08$ & $22.96 \pm 0.04$ & $261 \pm 100$ & $\ldots$ & \\
\hline $78: 56 \ldots \ldots \ldots \ldots$ & 33846.57 & -352513.1 & $2.68 \pm 0.01$ & $16.23 \pm 0.01$ & $43 \pm 26$ & $48 \pm 16$ & $76: 33$ \\
\hline $78: 60 \ldots \ldots \ldots . . .$. & 33845.47 & -352411.4 & $\ldots$ & $\ldots$ & $55 \pm 95$ & $\ldots$ & \\
\hline $78: 76 \ldots \ldots \ldots . .$. & 33841.08 & -352421.6 & $\ldots$ & $\ldots$ & $59 \pm 66$ & $146 \pm 40$ & \\
\hline 78:90 ................. & 33837.17 & -352520.4 & $0.53 \pm 0.01$ & $17.94 \pm 0.01$ & $90 \pm 52$ & $75 \pm 14$ & \\
\hline 80:47 ................. & 33819.84 & -352411.0 & $\ldots$ & $\ldots$ & $41 \pm 29$ & $33 \pm 16$ & \\
\hline $80: 94 \ldots \ldots \ldots . .$. & 33816.68 & -352131.9 & $\ldots$ & $\ldots$ & $31 \pm 68$ & $-54 \pm 33$ & \\
\hline $80: 99 \ldots \ldots \ldots . .$. & 33827.32 & $-3521 \quad 13.2$ & $2.71 \pm 0.02$ & $19.20 \pm 0.01$ & $-65 \pm 113$ & $-70 \pm 28$ & \\
\hline $80: 100 \ldots \ldots . . . .$. & 33827.32 & -352113.2 & $\ldots$ & $\ldots$ & $-39 \pm 60$ & $-72 \pm 24$ & \\
\hline $80: 105 \ldots \ldots \ldots . .$. & 33812.79 & -352057.4 & $\ldots$ & $\ldots$ & $71 \pm 51$ & $111 \pm 15$ & \\
\hline $81: 26 \ldots \ldots \ldots \ldots$ & 33806.77 & -352524.9 & $0.97 \pm 0.03$ & $21.20 \pm 0.03$ & $87 \pm 24$ & $197 \pm 29$ & \\
\hline $81: 28 \ldots \ldots \ldots \ldots . . .$. & 33804.21 & $-35 \quad 2515.1$ & $2.56 \pm 0.01$ & $19.37 \pm 0.01$ & $45 \pm 47$ & $14 \pm 16$ & \\
\hline $81: 38 \ldots \ldots \ldots \ldots . . .$. & 33802.11 & $-35 \quad 2440.2$ & $0.75 \pm 0.01$ & $18.95 \pm 0.01$ & $293 \pm 28$ & $290 \pm 13$ & \\
\hline 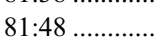 & 33808.82 & $-35 \quad 2400.4$ & $2.37 \pm 0.03$ & $18.91 \pm 0.02$ & $32 \pm 48$ & $30 \pm 17$ & \\
\hline 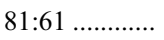 & 33815.02 & -352308.1 & $\ldots$ & $\ldots$ & $-1 \pm 87$ & $-12 \pm 36$ & \\
\hline $81: 102 \ldots \ldots \ldots . .$. & 33810.54 & -352037.8 & $1.98 \pm 0.01$ & $18.09 \pm 0.01$ & $-77 \pm 63$ & $-69 \pm 15$ & \\
\hline $82: 18 \ldots \ldots \ldots \ldots$ & 33802.10 & $-3524 \quad 40.2$ & $0.75 \pm 0.01$ & $18.95 \pm 0.01$ & $250 \pm 62$ & $243 \pm 8$ & $81: 38$ \\
\hline $82: 34 \ldots \ldots \ldots . .$. & 33808.04 & -352520.9 & $1.71 \pm 0.06$ & $16.85 \pm 0.06$ & $107 \pm 36$ & $95 \pm 15$ & \\
\hline $82: 63 \ldots \ldots \ldots . .$. & 33819.84 & -352411.1 & $1.72 \pm 0.01$ & $17.38 \pm 0.01$ & $89 \pm 24$ & $79 \pm 14$ & \\
\hline $84: 24 \ldots \ldots \ldots . .$. & 33848.12 & -353249.9 & $2.05 \pm 0.01$ & $18.53 \pm 0.01$ & $135 \pm 38$ & $121 \pm 17$ & \\
\hline $84: 43 \ldots . . . \ldots . . . .$. & 33854.75 & -353138.5 & $\ldots$ & $\ldots$ & $102 \pm 53$ & $93 \pm 25$ & \\
\hline $84: 46 \ldots \ldots \ldots . .$. & 33856.19 & -353126.3 & $1.26 \pm 0.07$ & $22.61 \pm 0.04$ & $-34 \pm 166$ & $6 \pm 36$ & \\
\hline $84: 47 \ldots \ldots \ldots \ldots$ & 33851.12 & -353121.3 & $3.09 \pm 0.01$ & $17.80 \pm 0.01$ & $104 \pm 91$ & $48 \pm 10$ & \\
\hline 84:51 ................ & 33855.88 & $\begin{array}{llll}-35 & 31 & 07.3\end{array}$ & $1.24 \pm 0.02$ & $20.82 \pm 0.02$ & $268 \pm 31$ & $185 \pm 23$ & \\
\hline 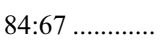 & 33849.44 & -353002.0 & $0.97 \pm 0.02$ & $20.49 \pm 0.01$ & $-49 \pm 49$ & $50 \pm 16$ & \\
\hline 84:82 ……............... & 33848.94 & -352910.1 & $2.19 \pm 0.01$ & $17.42 \pm 0.01$ & $\ldots$ & $81 \pm 10$ & \\
\hline 84:92 ................ & 33859.36 & -352834.9 & $3.01 \pm 0.01$ & $18.23 \pm 0.01$ & $\ldots$ & $-8 \pm 13$ & \\
\hline $86: 21 \ldots \ldots \ldots . .$. & 33831.28 & -353250.4 & $1.19 \pm 0.02$ & $20.90 \pm 0.01$ & $31 \pm 15$ & $9 \pm 17$ & \\
\hline $86: 32 \ldots \ldots \ldots \ldots$ & 33840.93 & -353207.6 & $3.12 \pm 0.01$ & $18.51 \pm 0.01$ & $\ldots$ & $6 \pm 23$ & \\
\hline
\end{tabular}


TABLE 4-Continued

\begin{tabular}{|c|c|c|c|c|c|c|c|}
\hline $\begin{array}{l}\text { Identifier } \\
\text { (1) }\end{array}$ & $\begin{array}{l}\text { R.A. (J2000.0) } \\
\text { (2) }\end{array}$ & $\begin{array}{c}\text { Decl. (J2000.0) } \\
\text { (3) }\end{array}$ & $\begin{array}{l}C-R \\
\text { (4) }\end{array}$ & $\begin{array}{c}R \\
(5)\end{array}$ & $\begin{array}{c}v_{c} \\
\left(\mathrm{~km} \mathrm{~s}^{-1}\right) \\
(6)\end{array}$ & $\begin{array}{c}v_{l} \\
\left(\mathrm{~km} \mathrm{~s}^{-1}\right) \\
(7)\end{array}$ & $\begin{array}{c}\text { Comments } \\
\text { (8) }\end{array}$ \\
\hline $86: 54 \ldots \ldots \ldots \ldots$ & 33833.24 & -353055.3 & $\ldots$ & $\ldots$ & $0 \pm 29$ & $-54 \pm 11$ & \\
\hline $89: 28 \ldots \ldots \ldots \ldots$ & 33824.70 & -353237.2 & $1.02 \pm 0.07$ & $22.73 \pm 0.05$ & $35 \pm 48$ & $-12 \pm 31$ & \\
\hline $90: 13 \ldots \ldots \ldots . . .$. & 33818.23 & -353333.5 & $3.16 \pm 0.01$ & $18.33 \pm 0.01$ & $-26 \pm 36$ & $-17 \pm 13$ & \\
\hline $90: 14 \ldots \ldots \ldots \ldots$ & 33808.02 & -353328.4 & $\ldots$ & $\ldots$ & $-21 \pm 23$ & $1 \pm 11$ & \\
\hline $90: 37 \ldots \ldots \ldots \ldots$ & 33818.38 & -353159.5 & $\ldots$ & $\ldots$ & $\ldots$ & $202 \pm 64$ & \\
\hline $90: 46 \ldots \ldots \ldots . . . .$. & 33813.19 & -353125.4 & $4.35 \pm 0.19$ & $19.76 \pm 0.13$ & $64 \pm 59$ & $\ldots$ & \\
\hline $90: 53 \ldots \ldots \ldots . . . .$. & 33809.91 & -353057.8 & $3.19 \pm 0.02$ & $18.78 \pm 0.01$ & $\ldots$ & $-35 \pm 35$ & \\
\hline 90:55 .............. & 33820.38 & -353049.4 & $1.23 \pm 0.04$ & $22.39 \pm 0.03$ & $67 \pm 80$ & $\ldots$ & \\
\hline 91:5 ….............. & 33807.43 & -353401.5 & $1.86 \pm 0.05$ & $21.90 \pm 0.03$ & $24 \pm 67$ & $\ldots$ & \\
\hline 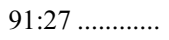 & 33804.96 & -353243.8 & $\ldots$ & $\ldots$ & $155 \pm 84$ & $136 \pm 29$ & \\
\hline 91:42 .............. & 33801.17 & -353145.3 & $2.93 \pm 0.03$ & $19.96 \pm 0.02$ & $84 \pm 74$ & $89 \pm 43$ & \\
\hline 91:77 ................. & 33801.12 & -352946.3 & $3.33 \pm 0.02$ & $18.90 \pm 0.01$ & $78 \pm 70$ & $\ldots$ & \\
\hline 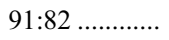 & 33758.35 & -352933.9 & $2.13 \pm 0.06$ & $21.43 \pm 0.04$ & $156 \pm 61$ & $\ldots$ & \\
\hline 91:97 ............... & 33805.03 & -352839.1 & $\ldots$ & $\ldots$ & $15 \pm 101$ & $\ldots$ & \\
\hline $92: 44 \ldots \ldots \ldots \ldots$ & 33810.78 & -352904.3 & $\ldots$ & $\ldots$ & $19 \pm 45$ & $-60 \pm 24$ & \\
\hline $92: 70 \ldots \ldots \ldots \ldots$ & 33801.73 & $\begin{array}{lll}-35 & 28 & 01.4\end{array}$ & $1.44 \pm 0.07$ & $22.23 \pm 0.03$ & $-348 \pm 34$ & $-141 \pm 32$ & \\
\hline $92: 80 \ldots \ldots \ldots \ldots$ & 33757.98 & -353101.1 & $\ldots$ & $\ldots$ & $\ldots$ & $15 \pm 19$ & \\
\hline 92:91 ............. & 33754.64 & -352755.6 & $1.58 \pm 0.01$ & $18.06 \pm 0.01$ & $39 \pm 21$ & $52 \pm 10$ & \\
\hline $92: 93 \ldots \ldots \ldots \ldots$ & 33753.95 & -35 2939.9 & $2.73 \pm 0.02$ & $19.50 \pm 0.01$ & $9 \pm 53$ & $20 \pm 23$ & \\
\hline $92: 57 \ldots \ldots \ldots \ldots$ & 33805.95 & -352850.4 & $\ldots$ & $\ldots$ & $2 \pm 35$ & $49 \pm 12$ & \\
\hline
\end{tabular}

${ }^{a}$ The meaning of the columns is the same as in Table 3. 
TABLE 5

Compilation of Clusters and Stars for which Velocities Have Previously Been Published IN THE Literature ${ }^{a}$

\begin{tabular}{|c|c|c|c|}
\hline Object & $\begin{array}{l}\text { Our Velocity } \\
\left(\mathrm{km} \mathrm{s}^{-1}\right)\end{array}$ & $\begin{array}{l}\text { Other Velocity } \\
\left(\mathrm{km} \mathrm{s}^{-1}\right)\end{array}$ & Other Denomination \\
\hline 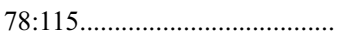 & $1358 \pm 34$ & $1150 \pm 59$ & Keck 19 \\
\hline $80: 19$ & $1266 \pm 30$ & $1260 \pm 66$ & Keck 14 \\
\hline 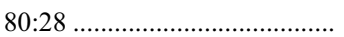 & $1753 \pm 18$ & $1688 \pm 42$ & Keck 18 \\
\hline 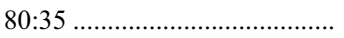 & $1536 \pm 21$ & $1523 \pm 30$ & Keck 15 \\
\hline 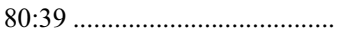 & $881 \pm 18$ & $866 \pm 42$ & Keck 17 \\
\hline 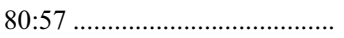 & $1309 \pm 27$ & $1374 \pm 126$ & Keck 20 \\
\hline 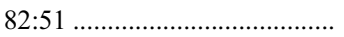 & $1933 \pm 75$ & $1732 \pm 42$ & Keck 12 \\
\hline 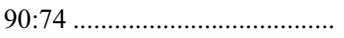 & $946 \pm 20$ & $732 \pm 32$ & Keck 1 \\
\hline 90:84 & $1292 \pm 20$ & $1094 \pm 34$ & Keck 2 \\
\hline 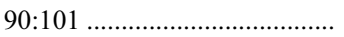 & $1559 \pm 18$ & $1386 \pm 31$ & Keck 6 \\
\hline 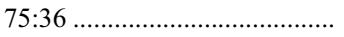 & $893 \pm 17$ & $994 \pm 73$ & NTT 203 \\
\hline $86: 9 \ldots \ldots \ldots$ & $1491 \pm 25$ & $1061 \pm 135$ & NTT 201 \\
\hline 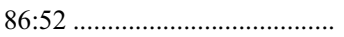 & $1395 \pm 18$ & $1440 \pm 138$ & NTT 113 \\
\hline 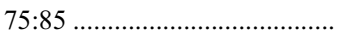 & $1609 \pm 24$ & $1742 \pm 150$ & AAT 57 \\
\hline 76:18 & $1803 \pm 33$ & $2026 \pm 150$ & AAT 49 \\
\hline 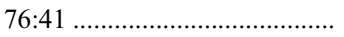 & $1031 \pm 34$ & $885 \pm 150$ & AAT 48 \\
\hline $76: 59 \ldots \ldots \ldots$ & $1652 \pm 32$ & $1941 \pm 150$ & AAT 54 \\
\hline …................... & $1654 \pm 19$ & $1623 \pm 150$ & AAT 43 \\
\hline 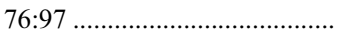 & $890 \pm 23$ & $1206 \pm 150$ & AAT 56 \\
\hline 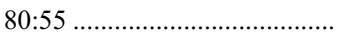 & $979 \pm 34$ & $1236 \pm 150$ & AAT 31 \\
\hline 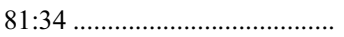 & $1230 \pm 34$ & $1784 \pm 150$ & AAT 17 \\
\hline 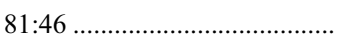 & $1837 \pm 31$ & $1921 \pm 150$ & AAT 27 \\
\hline 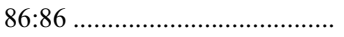 & $666 \pm 16$ & $541 \pm 150$ & AAT 41 \\
\hline 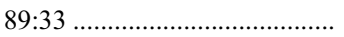 & $1615 \pm 34$ & $1859 \pm 150$ & AAT 30 \\
\hline 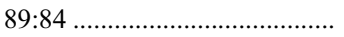 & $1708 \pm 13$ & $1836 \pm 150$ & AAT 20 \\
\hline $89: 93$ & $1329 \pm 30$ & $2182 \pm 150$ & AAT 25 \\
\hline 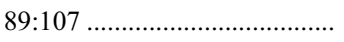 & $1170 \pm 39$ & $1646 \pm 150$ & AAT 26 \\
\hline 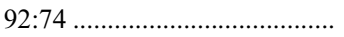 & $843 \pm 10$ & $1355 \pm 150$ & AAT 15 \\
\hline 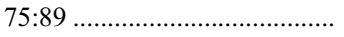 & $153 \pm 15$ & $97 \pm 45$ & $2 \mathrm{dF} 2889$ \\
\hline 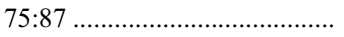 & $-31 \pm 22$ & $62 \pm 136$ & $2 \mathrm{dF} 3266$ \\
\hline 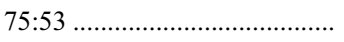 & $42 \pm 18$ & $116 \pm 29$ & $2 \mathrm{dF} 1770$ \\
\hline 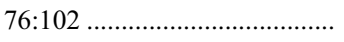 & $-7 \pm 18$ & $15 \pm 30$ & $2 \mathrm{dF} 3540$ \\
\hline 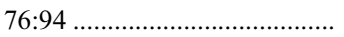 & $7 \pm 14$ & $45 \pm 55$ & $2 \mathrm{dF} 2889$ \\
\hline 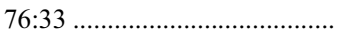 & $16 \pm 10$ & $300 \pm 55$ & $2 \mathrm{dF} 2755$ \\
\hline 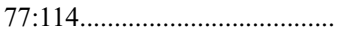 & $318 \pm 16$ & $287 \pm 43$ & $2 \mathrm{dF} 1610$ \\
\hline 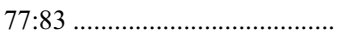 & $131 \pm 14$ & $189 \pm 84$ & $2 \mathrm{dF} 3001$ \\
\hline 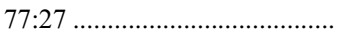 & $70 \pm 14$ & $171 \pm 146$ & $2 \mathrm{dF} 2574$ \\
\hline 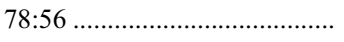 & $48 \pm 16$ & $85 \pm 35$ & $2 \mathrm{dF} 2756$ \\
\hline 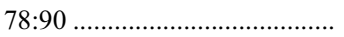 & $75 \pm 14$ & $171 \pm 146$ & $2 \mathrm{dF} 2574$ \\
\hline 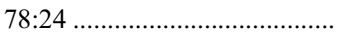 & $74 \pm 24$ & $115 \pm 29$ & $2 \mathrm{dF} 1770$ \\
\hline 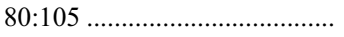 & $111 \pm 15$ & $124 \pm 11$ & $2 \mathrm{dF} 2048$ \\
\hline 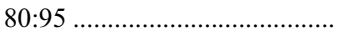 & $29 \pm 20$ & $127 \pm 41$ & $2 \mathrm{dF} 3133$ \\
\hline 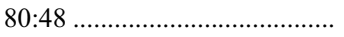 & $38 \pm 10$ & $63 \pm 85$ & $2 \mathrm{dF} 3353$ \\
\hline 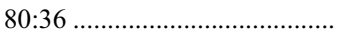 & $47 \pm 11$ & $158 \pm 38$ & $2 \mathrm{dF} 2569$ \\
\hline 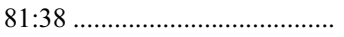 & $290 \pm 13$ & $195 \pm 58$ & $2 \mathrm{dF} 2530$ \\
\hline 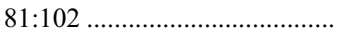 & $-69 \pm 15$ & $96 \pm 43$ & $2 \mathrm{dF} 869$ \\
\hline 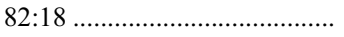 & $243 \pm 8$ & $195 \pm 57$ & $2 \mathrm{dF} 2530$ \\
\hline $82: 34$ & $95 \pm 15$ & $147 \pm 27$ & $2 \mathrm{dF} 2052$ \\
\hline 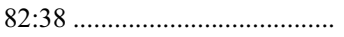 & $263 \pm 14$ & $435 \pm 45$ & $2 \mathrm{dF} 1407$ \\
\hline 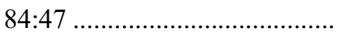 & $48 \pm 10$ & $191 \pm 51$ & $2 \mathrm{dF} 508$ \\
\hline 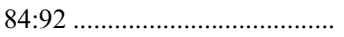 & $-8 \pm 13$ & $12 \pm 52$ & $2 \mathrm{dF} 1847$ \\
\hline 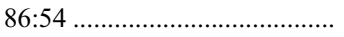 & $-42 \pm 11$ & $103 \pm 50$ & $2 \mathrm{dF} 3183$ \\
\hline 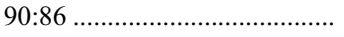 & $1233 \pm 9$ & $1312 \pm 57$ & $2 \mathrm{dF} 1254$ \\
\hline 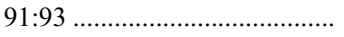 & $1233 \pm 9$ & $1312 \pm 57$ & $2 \mathrm{dF} 1254$ \\
\hline 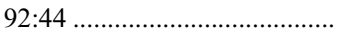 & $-60 \pm 24$ & $15 \pm 62$ & $2 \mathrm{dF} 3388$ \\
\hline 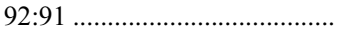 & $52 \pm 10$ & $55 \pm 82$ & $2 \mathrm{dF} 3146$ \\
\hline 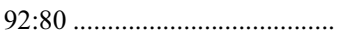 & $15 \pm 19$ & $-67 \pm 51$ & $2 \mathrm{dF} 3354$ \\
\hline
\end{tabular}

a The denomination references are as follows. Keck: Kissler-Patig et al. 1998; NTT: Minniti et al. 1998; AAT: Grillmair et al. 1994; 2dF: Drinkwater et al. 2001b. 
discrepancy probably is that we used line instead of correlation velocities for the stars, and we have shown earlier that the errors for the velocities obtained via line measurements are underestimated. These measurements are hence in good agreement with our velocities.
B. D., T. R., D. G., L. I., and D. M. gratefully acknowledge support from the Chilean Center for Astrophysics FONDAP No. 15010003. B.D. gratefully acknowledges financial support of the Alexander von Humboldt Foundation via a Feodor Lynen Stipendium.

\section{REFERENCES}

Arnaboldi, M., Freeman, K. C., Hui, X., Capaccioli, M., \& Ford, H. 1994, Messenger, 76, 40

Bertin, E., \& Arnouts, S. 1996, A\&AS, 117, 393

Bridges, T. J., Hanes, D. A., \& Harris, W. E. 1991, AJ, 101, 469

Cohen, J. G., \& Ryzhov, A. 1997, ApJ, 486, 230

Côté P., et al. 2001, ApJ, 559, 828

da Costa, L. N., et al. 1998, AJ, 116, 1

de Vaucouleurs, G., de Vaucouleurs, A., Corwin, H. G., Jr., Buta, R., Paturel, G., \& Fouqué, P. 1991, Third Reference Catalogue of Bright Galaxies (New York: Springer)

Dirsch, B., et al. 2003, AJ, 125, 1908 (Paper I)

Drinkwater, M., et al. 2001, AAO Newsl., 97, 9

Drinkwater, M. J., Gregg, M. D., Holman, B. A., \& Brown, M. J. I. 2001, MNRAS, 326, 1076

Gerhard, O., Kronawitter, A., Saglia, R. P., \& Bender, R. 2001, AJ, 121, 1936

Grillmair, C. J., Forbes, D. A., Brodie, J. P., \& Elson, R. A. W. 1999, AJ, 117,167

Grillmair, C. J., Freeman, K. C., Bicknell, G. V., Carter, D., Couch, W. J., Sommer-Larsen, J., \& Taylor, K. 1994, ApJ, 422, L9
Hanes, D. A., \& Harris, W. E. 1986, ApJ, 309, 564

Hanes, D. A., et al. 2001, ApJ, 559, 812

Kissler-Patig, M., et al. 1997, A\&A, 319, 470 1998, AJ, 115, 105 - 1999, AJ, 117, 1206

Kronawitter, A., Saglia, R. P., Gerhard O., \& Bender R. 2000, A\&AS, 144, 53 Merritt, D. 1993, ApJ, 413, 79

Merritt, D., \& Tremblay, B. 1993, AJ, 106, 2229

Minniti, D., Kissler-Patig M., Goudfrooij, P., \& Meylan, G. 1998, AJ, 115, 121 Napolitano, N. R., Arnaboldi, M., \& Capaccioli, M. 2002, A\&A, 383, 791

Ostrov, P. G., Geisler, D., \& Forte, J. C. 1993, AJ, 105, 1762

Richtler, T., et al. 2003, AJ, submitted (Paper II)

Saglia, R. P., Kronawitter, A., Gerhard, O., \& Bender, R. 2000, AJ, 119, 153 Tonry, J., \& Davis, M. 1979, AJ, 84, 1511

Urban, S. E., et al. 1998, AJ, 115, 1212

Wagner, S., Richtler, T., \& Hopp, U. 1991, A\&A, 241, 399 